Research Article

\title{
Damage Index Analysis of Retaining Wall Structures Based on the Impulse Response Function and Virtual Impulse Response Function
}

\author{
Qian Xu \\ School of Civil Engineering and Architecture, Shaanxi University of Technology, Hanzhong 723001, Shaanxi, China \\ Correspondence should be addressed to Qian Xu; xuqian20121601014@163.com
}

Received 9 April 2021; Revised 17 June 2021; Accepted 20 September 2021; Published 18 October 2021

Academic Editor: Jean-Jacques Sinou

Copyright (c) 2021 Qian Xu. This is an open access article distributed under the Creative Commons Attribution License, which permits unrestricted use, distribution, and reproduction in any medium, provided the original work is properly cited.

\begin{abstract}
To identify the damage within retaining wall structures, the Hilbert-Huang Transforms of the impulse response function and virtual impulse response function were performed. The Hilbert marginal energy ratio spectrums of the impulse response function and virtual impulse response function were acquired. To reflect damage information effectively, those bands with stronger damage sensitivity were extracted via the threshold value $\varepsilon_{0}$. Then, the Hilbert feature bands, which were more sensitive to damage within retaining walls, were selected by considering the contribution of the residual band to the damage identification. Based on the feature bands, the Hilbert damage feature vector, which reflects the variations of Hilbert marginal energy ratio caused by damage, was created. Based on the damage feature vector, two damage identification indexes (the energy ration standard deviation and Energy Ration Standard Deviation), which were based on the impulse response function and virtual impulse response function, respectively, were proposed to identify damage within retaining walls. To investigate the validity of the damage indexes, vibration tests on a pile plate retaining wall were done. The test results show that the damage feature vector is a zero vector or the value of damage index is zero when the wall is undamaged. The damage feature vector is a nonzero vector or the value of the damage index is more than zero when the wall is damaged. Thus, the damage state of the wall can be detected sensitively via the damage feature vector or damage indexes. Partial damage causes greater fluctuation of trend surface of the damage index. The location of partial damage can be diagnosed validly via the coordinate of peak value in the trend surface. The quantitative relationship formula between the damage index and damage intensity is established. The damage intensity of the wall can be calculated reversely, when the damage index is available. Either the energy ration standard deviation or Energy Ration Standard Deviation can be used to detect the damage state, diagnose the damage location, and identify the damage intensity. In comparison with the energy ration standard deviation, the stability and damage sensitivity of the Energy Ration Standard Deviation is much better.
\end{abstract}

\section{Introduction}

As a common type of retaining structure, the retaining walls (such as cantilever retaining walls, gravity retaining walls, and pile plate retaining walls) were often used to sustain foundation ditch, stabilize the cutting of highway, and prevent landslide or collapse. Thus, retaining walls were widely used in building engineering, road engineering, slope engineering, and underground engineering, and so on. However, under the effects of many factors (such as environmental erosion, material degradation, and load variation), such damages as micro-cracks or holes might appear in the retaining wall. The wall might collapse when these damages accumulated to a certain extent, which caused serious accidents, as listed in Table 1 (The data in Table 1 is from the Internet). To avoid or reduce these accidents in the future, it is necessary and significant to investigate the identification of damages of retaining wall structures.

Common damages within engineering structures mainly included internal faults, structural cracks, and holes. Changes of some structural parameters were often caused by these damages. Thus, the damaged state of structures could be detected, damage locations could be diagnosed, damage intensity could be identified, and residual lifespan could be 
TABLE 1: Accidents due to retaining walls collapse.

\begin{tabular}{|c|c|c|c|}
\hline Time & Place & Consequence & Cause \\
\hline 2012 & enwu city of Fujian pr & Destroyed many houses & ot found \\
\hline 2013 & $\begin{array}{c}\text { Shouyang city of Shanxi province, } \\
\text { China }\end{array}$ & 12 deaths and 7 & $\begin{array}{c}\text { Damages were not paid much attention to even after detecting } \\
\text { them }\end{array}$ \\
\hline 2014 & $\begin{array}{c}\text { Qingdao city of Shandong province, } \\
\text { China }\end{array}$ & $18 \mathrm{~d}$ & Damages caused by rainfall cau \\
\hline 2015 & $\begin{array}{c}\text { Lanling city of Shandong province, } \\
\text { China }\end{array}$ & 10 deaths & Constructio \\
\hline 2016 & $\begin{array}{r}\text { Quanzhou city of } \\
\text { Chi }\end{array}$ & 2 deaths & Rainfall and construction qu \\
\hline 2017 & $\begin{array}{c}\text { Chizhou city of Anhui province, } \\
\text { China }\end{array}$ & . & Construction quality faults caused the \\
\hline 2018 & aoding city of $\mathrm{He}$ & & \\
\hline 2019 & Bijie city of Guizhou province, China & & \\
\hline 2020 & $\begin{array}{c}\text { Jincheng city of Shanxi province, } \\
\text { China }\end{array}$ & 3 & $\begin{array}{l}\text { Rainfall and construction quality faults caused the collapse of } \\
\text { the wall }\end{array}$ \\
\hline
\end{tabular}

evaluated by analyzing these structural parameters [1]. And, common methods for damage identification are listed here. (1) The methods were based on such dynamic fingerprints as natural frequencies, mode shapes, and frequency response function, Hearn and Testa identified the damages within a steel frame and wire rope via analyzing the changes of natural frequencies [2]. Biswas et al. detected the damage intensity and location of the highway bridge via analysis of the mode shapes [3]. (2) The methods based on the finite element model updating. Zhang et al. diagnosed the damages within structures via synchronically updating measured vibration modes and finite element model [4]. Zong et al. proposed a damage diagnosis method for bridges based on the response surface model updating and the element modal strain energy [5]. (3) The methods based on the genetic algorithm. Perera and Torres localized the damage within a beam via the analysis of natural frequencies and mode shapes based on the genetic algorithm [6]. Varmazyar et al. detected the damage within beams via analysis of the response power spectral density of the beam based the evolutionary algorithms [7]. (4) The method based on the neural network. Qu and Chen proposed a seismic damage diagnosis method for structures based on the neural network system of damage diagnosis [8]. Wang et al. proposed a new damage identification method based on the statistical neural network [9]. (5) The methods based on the dynamic responses. Hou et al. diagnosed damages within the Benchmark structure via the Wavelet Packet Decomposition of structural vibration signals [10]. Based on the extracted impulse response function, Li and Fan detected damages within a beam via optimizing sensor locations [11]. Ding et al. proposed a damage alarming method for structures based on the Wavelet Packet Decomposition of the virtual impulse response function [12]. Zheng et al. proposed a damage diagnosis method for bearings based on the Hilbert-Huang Transform of vibration signals to detect faults in bearings [13].

The damage identification methods based on structural dynamic fingerprints were widely used to diagnose damages, because these fingerprints could be easily acquired. However, it was easy to obtain the damage information of bands with lower frequencies but hard to measure the ones with higher frequencies via this method due to the limitation of existing measurement technology. Some damage information might be lost via this method. The method based on the finite element model updating required better environments (fewer interferences such as size effects, variation of temperature, and humidity) and excitations. However, the better environments and sufficient excitations were rare in practical engineering. Thus, this method might not be suitable for practical engineering with pool conditions and several interferences. The damage identification methods based on the genetic algorithm and neural network were affected greatly by sample functions, objective functions, and algorithms. The precision of damage identification was greatly dependent on these factors. The method based on the Wavelet Packet Transform had much better damage sensitivity and stronger noise immunity. However, the method lacked adaptivity to signals and was suitable only for linear signals. In addition, the wavelet packet analysis results were greatly dependent on the wavelet basis function. In comparison with the WPT (the Wavelet Packet Transform), the method based on HHT (the Hilbert-Huang Transform) had good adaptivity to signals and was suitable for either linear signals or nonlinear ones.

Thus far, the attention of damage identification to engineering structures were mainly paid to such structural components as beams [14], plates [15], piles [16], building structures $[17,18]$, bridge structures $[19,20]$, and other structures [21, 22]. However, the investigation of damage identification to retaining wall structures was much rarer. Consequently, the damage identification to retaining wall structures should be paid much attention. In this paper, the Hilbert marginal energy ratio spectrums of the impulse response function and virtual impulse response function are acquired via HHT. Then, the Hilbert feature bands, which are more sensitive to damage within retaining walls, are selected by considering the contribution of the residual band to the damage diagnosis. Based on the feature bands, the Hilbert damage feature vector, which reflects the variations of Hilbert marginal energy ratio caused by damage, is created. Based on the damage feature vector, two damage 
identification indexes (the energy ration standard deviation and Energy Ration Standard Deviation), which are based on the impulse response function and virtual impulse response function, are proposed to identify damage within retaining walls. To investigate the validity of the damage indexes, vibration tests on a pile plate retaining wall are done. The damage within the retaining wall is identified via the energy ration standard deviation and Energy Ration Standard Deviation.

\section{Damage Identification Indexes}

In the light of literature [23, 24], damages within the structure cannot be identified validly, if the dynamic responses to the structure are analyzed directly via WPT or HHT. However, damages within the structure can be identified validly based on IRF (the Impulse Response Function of the structural response and external excitation in a structure) or VIRF (the Virtual Impulse Response Function of the virtual response and virtual excitation in a structure).

2.1. The Impulse Response Function. Under the effects of excitation, the motion equation of a multiple degree of freedom structural dynamic system can be expressed as

$$
\mathbf{m} \ddot{\mathbf{u}}+\mathbf{c} \dot{\mathbf{u}}+\mathbf{k u}=\mathbf{f}(t)
$$

where $\mathbf{m} \in \mathbf{R}^{n \times n}$ is the mass matrix of the structural dynamic system, $\mathbf{c} \in \mathbf{R}^{n \times n}$ is the damping matrix, $\mathbf{k} \in \mathbf{R}^{n \times n}$ is the stiffness matrix, $\mathbf{u} \in \mathbf{R}^{n \times 1}$ is the displacement matrix, and $\mathbf{f} \in \mathbf{R}^{n \times 1}$ is the excitation matrix.

The Fourier Transforms of the excitation $\mathbf{f}(t)$ and response $\mathbf{u}(t)$ are performed, respectively:

$$
\begin{aligned}
& \mathbf{u}(\omega)=\int_{-\infty}^{\infty} e^{-i \omega t} \mathbf{u}(t) \mathrm{d} t \\
& \mathbf{f}(\omega)=\int_{-\infty}^{\infty} e^{-i \omega t} \mathbf{f}(t) \mathrm{d} t
\end{aligned}
$$

where $\mathbf{u}(\omega)$ and $\mathbf{f}(\omega)$ are the Fourier Transforms of $\mathbf{u}(t)$ and f $(t)$, respectively.

Then, the frequency response function $\mathbf{h}(\omega)$ of $\mathbf{u}(t)$ and $\mathbf{f}$ $(t)$ can be obtained and expressed as

$$
\mathbf{h}(\omega)=\frac{\mathbf{u}(\omega)}{\mathbf{f}(\omega)}
$$

After the inverse Fourier Transforms of $\mathbf{h}(\omega)$, IRF $\mathbf{h}(t)$ is obtained and expressed as

$$
\mathbf{h}(t)=\frac{1}{2 \pi} \int_{-\infty}^{\infty} e^{-i \omega t} \mathbf{h}(\omega) \mathrm{d} \omega
$$

2.2. The Virtual Impulse Response Function. Under the effects of excitation $\mathbf{F}(t)$, the dynamic response at a reference point can be regarded as a virtual excitation, and the response at a calculating point can be regarded as a virtual response. VIRF can be acquired via the virtual excitation and virtual response. The auto-power spectral density $\mathbf{G}_{u u}(\omega, i, j)$ of the virtual excitation $U_{j}(t)$ (the dynamic response at the reference point $j$ ) can be expressed as

$$
\mathbf{G}_{u u}(\omega, j)=\mathbf{U}^{*}(\omega, j) \cdot \mathbf{U}(\omega, j),
$$

where $\mathbf{U}_{j}(\omega, j)$ is the Fourier Transform of the $\mathbf{U}_{j}(t)$ and $\mathbf{U} *(\omega, j)$ is the complex conjugate of $\mathbf{U}_{j}(t)$.

The cross-power spectral density $\mathbf{G}_{y u}(\omega, i, j)$ between $\mathbf{U}_{j}$ $(t)$ and the virtual response $\mathbf{U}_{i}(t)$ (the response at the calculating point $i$ ) can be expressed as

$$
\mathbf{G}_{\mathrm{yu}}(\omega, i, j)=\mathbf{U}^{*}(\omega, j) \cdot \mathbf{U}(\omega, i),
$$

where $\mathbf{U}(\omega, i)$ is the Fourier Transform of $\mathbf{U}_{i}(t)$.

$\mathbf{U}(\omega, j)$ and $\mathbf{U}(\omega, i)$ can be, respectively, expressed as

$$
\begin{aligned}
& \mathbf{U}(\omega, j)=\mathbf{H}(\omega, j) \mathbf{F}(\omega), \\
& \mathbf{U}(\omega, i)=\mathbf{H}(\omega, i) \mathbf{F}(\omega),
\end{aligned}
$$

and the virtual frequency response function $\mathbf{H}_{y u}(\omega, i, j)$ of $\mathbf{U}_{j}$ $(t)$ and $\mathbf{U}_{i}(t)$ can be expressed as

$$
\begin{aligned}
\mathbf{H}_{\mathrm{yu}}(\omega, i, j) & =\frac{\mathbf{G}_{\mathrm{yu}}(\omega, i, j)}{\mathbf{G}_{\mathrm{uu}}(\omega, j)}=\frac{\mathbf{U}^{*}(\omega, j) \cdot \mathbf{U}(\omega, i)}{\mathbf{U}^{*}(\omega, j) \cdot \mathbf{U}(\omega, j)} \\
& =\frac{\left(\mathbf{H}^{*}(\omega, j) \mathbf{F}^{*}(\omega)\right) \cdot(\mathbf{H}(\omega, i) \mathbf{F}(\omega))}{\left(\mathbf{H}^{*}(\omega, j) \mathbf{F}^{*}(\omega)\right) \cdot(\mathbf{H}(\omega, j) \mathbf{F}(\omega))} \\
& =\frac{\mathbf{H}^{*}(\omega, j) \cdot \mathbf{H}(\omega, i)}{\mathbf{H}^{*}(\omega, j) \cdot \mathbf{H}(\omega, j)} .
\end{aligned}
$$

After the inverse Fourier Transform of $\mathbf{H}_{y u}(\omega, i, j)$, VIRF $\mathbf{H}_{y u}(t, i, j)$ of $\mathbf{U}_{j}(t)$ and $\mathbf{U}_{i}(t)$ can be expressed as

$$
\mathbf{H}_{\mathrm{yu}}(t, i, j)=\frac{1}{2 \pi} \int_{-\infty}^{\infty} e^{-i \omega t} \mathbf{H}(\omega, i, j) \mathrm{d} \omega .
$$

From the analysis above, both IRF and VIRF are the inherent attributes of the structure. IRF can be acquired when both the external excitation and structural responses are available. In comparison with IRF, VIRF can be acquired when the virtual response and virtual excitation are available whether the external excitation is available or not. Thus, VIRF has no dependence on the external excitation.

2.3. Damage Identification Indexes. As mentioned before, damages within engineering structures can be identified based on IRF or VIRF via WPT or HHT. However, WPT lacks adaptivity to signals and is only suitable for linear signals. And, the wavelet packet analysis results are greatly dependent on the wavelet basis function. In comparison with WPT, HHT has good adaptivity to signals, and HHT is suitable for either linear signals or nonlinear ones [25]. Thus, the Hilbert-Huang Transform of IRF and VIRF are performed, respectively. Then, the intrinsic mode functions of IRF and VIRF are acquired, respectively. On the basis of the Hilbert marginal energy ratio spectrum, the Hilbert feature vectors, which are based on IRF and VIRF, are created, respectively. Based on the Hilbert feature vectors, the Hilbert damage feature vectors are created, respectively. On the basis 
of the damage feature vectors, two damage identification indexes are proposed.

To obtain intrinsic mode functions, the empirical mode decompositions of IRF $h(t)_{u}$ and $h(t)_{d}$ are performed, respectively. And, the empirical mode decompositions of VIRF $H(t)_{u}$ and $H(t)_{d}$ are performed too, respectively. The $h$ $(t)_{u}, h(t)_{d}, H(t)_{u}$, and $H(t)_{d}$ can be expressed as

$$
\begin{aligned}
& \left\{\begin{array}{l}
h(t)_{u}=\left\{\mathrm{imf}_{u}=\operatorname{imf}_{1 u}+\mathrm{imf}_{2 u}+\cdots+\mathrm{imf}_{\mathrm{un}}+r(t)_{\mathrm{nu}},\right. \\
H(t)_{u}=\{\mathrm{IMF}\}_{u}=\mathrm{IMF}_{1 u}+\mathrm{IMF}_{2 u}+\cdots+\mathrm{IMF}_{\mathrm{nu}}+R(t)_{\mathrm{nu}},
\end{array}\right. \\
& \left\{\begin{array}{l}
h(t)_{d}=\{\mathrm{imf}\}_{d}=\mathrm{imf}_{1 d}+\mathrm{imf}_{2 d}+\cdots+\mathrm{imf}_{\mathrm{ud}}+r(t)_{\mathrm{nd}}, \\
H(t)_{d}=\left\{\mathrm{IMF}_{d}=\mathrm{IMF}_{1 d}+\mathrm{IMF}_{2 d}+\cdots+\mathrm{IMF}_{\mathrm{nd}}+R(t)_{\mathrm{nd}},\right.
\end{array}\right.
\end{aligned}
$$

where the $h(t)_{u}$ and $h(t)_{d}$ are IRFs of the undamaged retaining wall and the damaged one, respectively. The $H(t)_{u}$ and $H(t)_{d}$ are VIRFs of the undamaged wall and damaged one, respectively. The $\{\mathrm{imf}\}_{u}$ and $\{\mathrm{imf}\}_{d}$ are the intrinsic mode functions of the $h(t)_{u}$ and $h(t)_{d}$, respectively. The $\{\mathrm{IMF}\}_{u}$ and $\{\mathrm{IMF}\}_{d}$ are the intrinsic mode functions of the $H$ $(t)_{u}$ and $H(t)_{d}$, respectively. The $r(t)_{n u}, r(t)_{n d}, R(t)_{n u}$, and $R$ $(t)_{n d}$ are residual functions after the empirical mode decomposition of $h(t)_{u}, h(t)_{d}, H(t)_{u}$, and $H(t)_{d}$, respectively. And, the $n$ is the order of empirical mode decomposition [26].

Because the values of residual functions are much less, the residual functions can be neglected. Thus, the $h(t)_{u}, h$ $(t)_{d}, H(t)_{\mathcal{u}}$, and $H(t)_{d}$ can be rewritten as:

$$
\begin{aligned}
& \left\{\begin{array}{l}
h(t)_{u}=\{\mathrm{imf}\}_{u}=\operatorname{imf}_{1 u}+\operatorname{imf}_{2 u}+\cdots+\operatorname{imf}_{\mathrm{un}}, \\
H(t)_{u}=\{\mathrm{IMF}\}_{u}=\mathrm{IMF}_{1 u}+\mathrm{IMF}_{2 u}+\cdots+\mathrm{IMF}_{\mathrm{nu}},
\end{array}\right. \\
& \left\{\begin{array}{l}
h(t)_{d}=\{\mathrm{imf}\}_{d}=\operatorname{imf}_{1 d}+\operatorname{imf}_{2 d}+\cdots+\mathrm{imf}_{\mathrm{ud}}, \\
H(t)_{d}=\{\mathrm{IMF}\}_{d}=\mathrm{IMF}_{1 d}+\mathrm{IMF}_{2 d}+\cdots+\mathrm{IMF}_{\mathrm{nd}} .
\end{array}\right.
\end{aligned}
$$

In the light of the empirical mode decomposition [26], the components of intrinsic mode functions $\operatorname{imf}_{j u}, \operatorname{imf}_{j d}$, $\operatorname{IMF}_{j u}$, and $\operatorname{IMF}_{j d}(j=1,2, \ldots, n)$ are expressed as:

$$
\begin{aligned}
& \left\{\begin{array}{l}
\operatorname{imf}_{j u}=\operatorname{Re}\left[\bar{\alpha}_{j u}(t) \cdot e^{i \int \bar{\omega}_{j \mathrm{u}}(t) \mathrm{d} t}\right], \\
\operatorname{IMF}_{j u}=\operatorname{Re}\left[\alpha_{j u}(t) \cdot e^{i \int \omega_{\mathrm{ju}}(t) \mathrm{d} t}\right],
\end{array}\right. \\
& \left\{\begin{array}{l}
\operatorname{imf}_{j d}=\operatorname{Re}\left[\bar{\alpha}_{j d}(t) \cdot e^{i \int \bar{\omega}_{\mathrm{jd}}(t) \mathrm{d} t}\right], \\
\operatorname{IMF}_{j d}=\operatorname{Re}\left[\alpha_{j d}(t) \cdot e^{i \int \omega_{\mathrm{jd}}(t) \mathrm{d} t}\right],
\end{array}\right.
\end{aligned}
$$

where the $\bar{a}_{j}(t)$ and $e^{i \int \bar{\omega}_{j}(t) \mathrm{d} t}$ are the instantaneous amplitude and instantaneous frequency after the empirical mode decomposition of IRF, the $a_{j}(t)$ and $e^{i \int \omega_{j}(t) \mathrm{d} t}$ are the instantaneous amplitude and instantaneous frequency after the empirical mode decomposition of VIRF. Re means real part.

Then, the Hilbert spectrums $\mathbf{h}(\bar{\omega}, t)_{u}$ and $\mathbf{h}(\bar{\omega}, t)_{d}$ are acquired via the Hilbert Transformation of the intrinsic mode functions $\left\{\mathrm{imf}_{u}\right.$ and $\{\mathrm{imf}\}_{d}$. The Hilbert spectrums $\mathbf{H}$ $(\omega, t)_{u}$ and $\mathbf{H}(\omega, t)_{d}$ are acquired via $\{\mathrm{IMF}\}_{u}$ and $\{\mathrm{IMF}\}_{d}$, as expressed in (13) and (14).

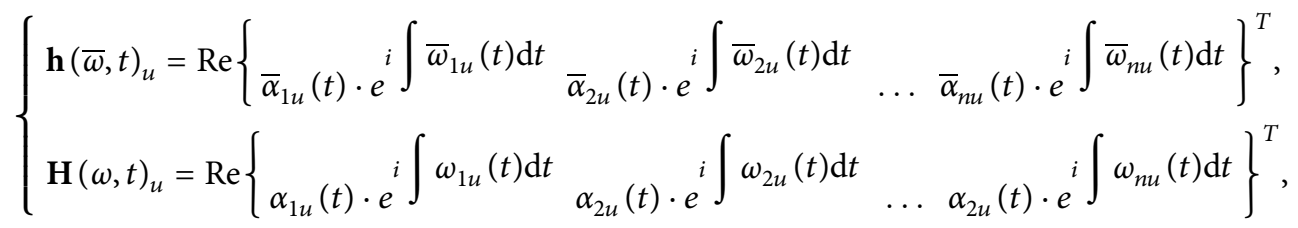

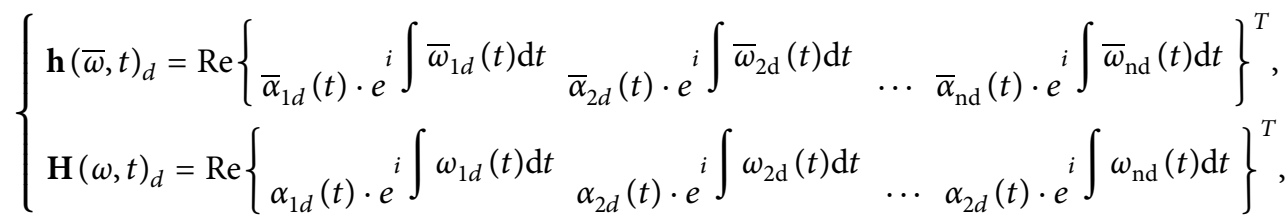

where $\bar{\omega}_{j}<\bar{\omega}_{j+1}, \omega_{j}<\omega_{j+1}, j=1,2, \ldots, n-1$.

Based on the $\mathbf{h}(\bar{\omega}, t)_{u}, \mathbf{h}(\bar{\omega}, t)_{d}, \mathbf{h}(\omega, t)_{u}$, and $\mathbf{H}(\omega, t)_{d}$, the Hilbert marginal energy spectrums $\mathbf{e}(\bar{\omega})_{u}, \mathbf{e}(\bar{\omega})_{d}, \mathbf{E}(\omega)_{u}$, and $\mathbf{E}(\omega)_{d}$ can be expressed as $[27,28]$ : 


$$
\begin{aligned}
& \left\{\begin{array}{l}
\mathbf{e}(\bar{\omega})_{u}=\left[\begin{array}{llll}
e(\bar{\omega})_{1 u} & e(\bar{\omega})_{2 u} & \ldots & e(\bar{\omega})_{n u}
\end{array}\right]^{\mathrm{T}}=\left[\begin{array}{llll}
\int_{0}^{t} h(\bar{\omega})_{u} \mathrm{~d} t & \int_{0}^{t} h\left(\bar{\omega}_{2}, t\right)_{u} \mathrm{~d} t & \ldots \int_{0}^{t} h\left(\bar{\omega}_{n}, t\right)_{u} \mathrm{~d} t
\end{array}\right]^{T}, \\
\mathbf{e}(\bar{\omega})_{\mathrm{d}}=\left[\begin{array}{lllll}
e(\bar{\omega})_{1 d} & e(\bar{\omega})_{2 d} & \ldots & e(\bar{\omega})_{n d}
\end{array}\right]^{T}=\left[\begin{array}{llll}
\int_{0}^{t} h\left(\bar{\omega}_{1}, t\right)_{d} \mathrm{~d} t \int_{0}^{t} h\left(\bar{\omega}_{2}, t\right)_{d} \mathrm{~d} t & \ldots & \int_{0}^{t} h\left(\bar{\omega}_{n}, t\right)_{d} \mathrm{~d} t
\end{array}\right]^{T},
\end{array}\right.
\end{aligned}
$$

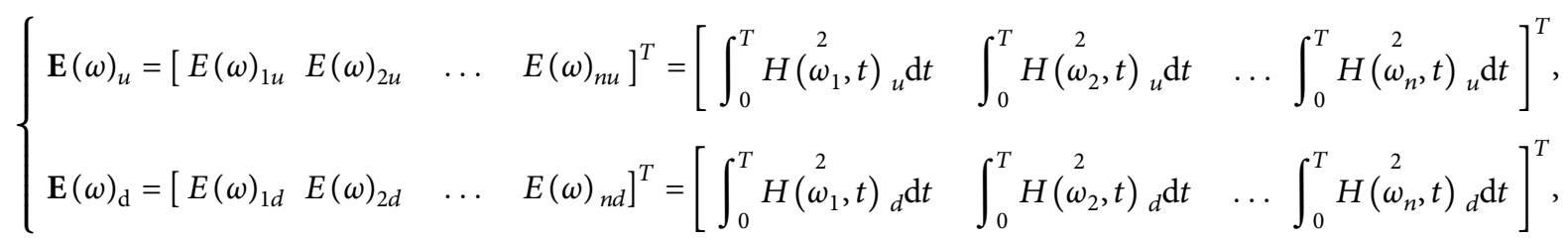

where the $\mathbf{e}(\bar{\omega})_{u}$ and $\mathbf{e}(\bar{\omega})_{d}$ are the Hilbert marginal energy spectrums of the $h(t)_{u}$ and $h(t)_{d}$, the $\mathbf{E}(\omega)_{u}$ and $\mathbf{E}(\omega)_{d}$ are the Hilbert marginal energy spectrums of the $H(t)_{u}$ and $H$ $(t)_{d}$, the $t$ and $T$ are time lengths of the signal.

The distribution characteristics of the signal energy can be reflected via the Hilbert marginal energy from an absolute energy perspective. However, the distribution characteristics of the signal energy are often reflected from such relative energy perspective as the Hilbert marginal energy ratio. Then, the Hilbert marginal energy ratio $\operatorname{er}(\bar{\omega})_{j u}, \operatorname{er}(\bar{\omega})_{j \mathrm{dd}}$, ER $(\omega)_{j u}$, and ER $(\omega)_{j u}$ can be defined as

$$
\begin{aligned}
& \left\{\begin{array}{l}
\operatorname{er}(\bar{\omega})_{j u}=\frac{e(\bar{\omega})_{j u}}{\sum_{j=1}^{n} e(\bar{\omega})_{j u}} \times 100, \\
\operatorname{er}(\bar{\omega})_{j d}=\frac{e(\bar{\omega})_{j d}}{\sum_{j=1}^{n} e(\bar{\omega})_{j d}} \times 100
\end{array}\right. \\
& \left\{\begin{array}{l}
\operatorname{ER}(\omega)_{j u}=\frac{E(\omega)_{j u}}{\sum_{j=1}^{n} E(\omega)_{j u}} \times 100 \\
\operatorname{ER}(\omega)_{j d}=\frac{E(\omega)_{j d}}{\sum_{j=1}^{n} E(\omega)_{j d}} \times 100
\end{array}\right.
\end{aligned}
$$

where the $e(\bar{\omega})_{\mathrm{ju}}$ and $e(\bar{\omega})_{\mathrm{jd}}$ are the Hilbert marginal energies of the $h(t)_{u}$ and $h(t)_{d}$, the $E(\omega)_{j u}$ and $E(\omega)_{j d}$ are the Hilbert marginal energies of the $H(t)_{u}$ and $H(t)_{d}$.
Then, the Hilbert marginal energy ratio spectrums $\operatorname{er}(\bar{\omega})_{u}, \operatorname{er}(\bar{\omega})_{d}$, ER $(\omega)_{u}$, and ER $(\omega)_{d}$ can be expressed as

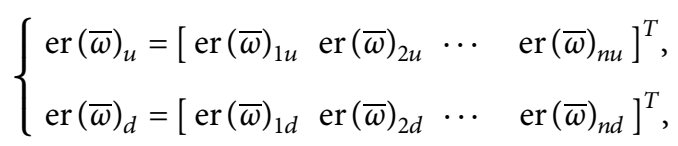

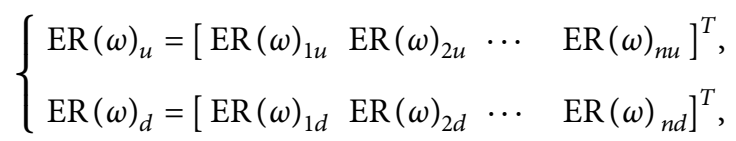

where $\operatorname{er}(\bar{\omega})_{u}$ and $\operatorname{er}(\bar{\omega})_{d}$ are the Hilbert marginal energy ratio spectrums of the $h(t)_{u}$ and $h(t)_{d}$, respectively. The ER $(\omega)_{u}$ and ER $(\omega)_{d}$ are the Hilbert marginal energy ratio spectrums of the $H(t)_{u}$ and $H(t)_{d}$, respectively.

In the literature [29], due to the influence of inherent frequencies of the retaining wall, not all the sub-bands are useful for damage identification. Only those bands, whose frequencies are close to the ones of the retaining wall, are more useful for damage identification. In this paper, to sensitively identify damages within the wall, let $\varepsilon_{\mathrm{IRF}}=\operatorname{er}(\bar{\omega})_{\mathrm{ju}}-\operatorname{er}(\bar{\omega})_{\mathrm{jd}} /\left|\operatorname{er}(\bar{\omega})_{\mathrm{ju}}-\operatorname{er}(\bar{\omega})_{\mathrm{jd}}\right|_{\max } i=1,2, \ldots, n$, $\varepsilon_{\mathrm{VIRF}}=\left|\operatorname{ER}(\omega)_{\mathrm{ju}}-\operatorname{ER}(\omega)_{\mathrm{jd}}\right| /\left|\operatorname{ER}(\omega)_{\mathrm{ju}}-\operatorname{ER}(\omega)_{\mathrm{jd}}\right|_{\max }$, where 
$\varepsilon_{\text {IRF }}$ and $\varepsilon_{\text {VIRF }}$ are the ratios of marginal energy ratio deviations of $\operatorname{er}(\bar{\omega})$ and ER $(\omega)$, respectively. To extract damage information, a threshold value $\varepsilon_{0}$ is defined, where $\varepsilon\left(\varepsilon_{\text {IRF }}\right.$ or $\varepsilon_{\text {VIRF }} \geq \varepsilon_{0}$. Then, the $p(p \leq n)$ bands can be selected from the $n$ bands via $\varepsilon_{0}$. These energy ratios of the $p$ bands can be used to reflect damage information. However, the contribution of the residual $(n-p)$ sub-bands to damage identification should not be ignored either. Thus, the $(n-p)$ sub-bands are merged into one band, which is called the residual band. Then, the energy ratios of the residual bands are defined as $\operatorname{er}(\bar{\omega})_{p+1} \quad$ and $\operatorname{ER}(\omega)_{p+1}$. Here, $\operatorname{er}(\bar{\omega})_{(p+1) u}=100-\sum_{q=1}^{p} \operatorname{er}(\bar{\omega})_{\mathrm{qu}} \quad$ and $\operatorname{er}(\bar{\omega})_{(p+1) \mathrm{d}}=100-\sum_{q=1}^{p} \operatorname{er}(\bar{\omega})_{\mathrm{qd}}$ are the energy ratios of the residual band of the $h(t)_{u}$ and $h(t)_{d}$, respectively. Similarly, $\operatorname{ER}(\omega)_{(p+1) u}=100-\sum_{q=1}^{p} \operatorname{ER}(\omega)_{\mathrm{qu}} \quad$ and $\operatorname{ER}(\omega)_{(p+1) d}=100-\sum_{q=1}^{p} \operatorname{ER}(\omega)_{\mathrm{qd}}$ are the energy ratios of the residual bands of the $H(t)_{u}$ and $H(t)_{d}$, respectively.

The $(p+1)$ bands are defined as feature bands. The feature bands are sorted from the strongest damage sensitivity to the poorest one. Then, the Hilbert feature $\operatorname{vectorserv}(\bar{\omega})_{\mathcal{u}}$, erv $(\bar{\omega})_{\mathrm{d}}, \mathbf{E R V}(\omega)_{u}$, and ERV $(\omega)_{d}$ can be obtained and expressed as

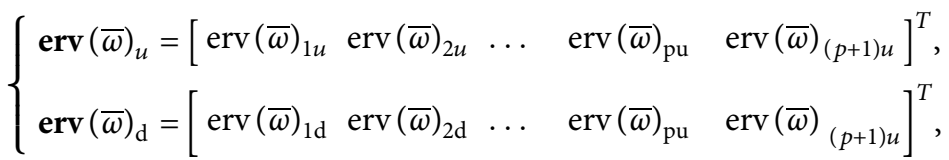

$$
\begin{aligned}
& \left\{\begin{array}{lllll}
\operatorname{ERV}(\omega)_{u} & =\left[\begin{array}{lllll}
\operatorname{ERV}(\omega)_{1 u} & \operatorname{ERV}(\omega)_{2 u} & \cdots & \operatorname{ERV}(\omega)_{\mathrm{pu}} & \operatorname{ERV}(\omega)_{(p+1) u}
\end{array}\right]^{T}, \\
\operatorname{ERV}(\omega)_{\mathrm{d}}=\left[\begin{array}{lllll}
\operatorname{ERV}(\omega)_{1 \mathrm{~d}} & \operatorname{ERV}(\omega)_{2 \mathrm{~d}} & \cdots & \operatorname{ERV}(\omega)_{\mathrm{pu}} & \operatorname{ERV}(\omega)_{(p+1) u}
\end{array}\right]^{T},
\end{array}\right.
\end{aligned}
$$

where the $\operatorname{erv}(\bar{\omega})_{k u}$ and $\operatorname{erv}(\bar{\omega})_{k d}(k=1,2, \ldots, p, p+1)$ are the energy ratios of the $k^{\text {th }}$ feature band in $\operatorname{erv}(\bar{\omega})_{u}$ and $\operatorname{erv}(\bar{\omega})_{d}$, respectively. ERV $(\omega)_{k u}$ and ERV $(\omega)_{k d}$ are the energy ratios of the $k^{\text {th }}$ feature band in ERV $(\omega)_{u}$ and ERV $(\omega)_{d}$, respectively.

On the basis of the $\operatorname{erv}(\bar{\omega})_{u}, \operatorname{erv}(\bar{\omega})_{d}, \operatorname{ERV}(\omega)_{u}$, and ERV $(\omega)_{d}$, the Hilbert marginal energy ratio deviation $\operatorname{erd}(\bar{\omega})_{k}$ and $\operatorname{ERD}(\omega)_{k}$ are calculated and expressed as

$$
\left\{\begin{array}{l}
\operatorname{erd}(\bar{\omega})_{k}=\left|\operatorname{erv}(\bar{\omega})_{k u}-\operatorname{erv}(\bar{\omega})_{k d}\right|, \\
\operatorname{ERD}(\omega)_{k}=\left|\operatorname{ERV}(\omega)_{k u}-\operatorname{ERV}(\omega)_{k d}\right|
\end{array}\right.
$$

If the energy ratios in the $\operatorname{erv}(\bar{\omega})_{u}$ can be regarded as baselines, the energy ratios in the erv $(\bar{\omega})_{d}$ fluctuate up and down on the basis of the ones in $\operatorname{erv}(\bar{\omega})_{u}$ due to damages. Similarly, energy ratios in the ERV $(\omega)_{d}$ also fluctuate up and down on the basis of the ones in ERV $(\omega)_{u}$.

To describe the fluctuation intensity of energy ratios, the Hilbert damage feature vectors erd $(\bar{\omega})_{d}$ and ERD $(\omega)$ are acquired and expressed as

$$
\left\{\begin{array}{l}
\operatorname{erd}(\bar{\omega})=\left[\begin{array}{lllll}
\operatorname{erd}(\bar{\omega})_{1} & \operatorname{erd}(\bar{\omega})_{2} & \ldots & \operatorname{erd}(\bar{\omega})_{p} & \operatorname{erd}(\bar{\omega})_{p+1}
\end{array}\right]^{T}, \\
\operatorname{ERD}(\omega)=\left[\begin{array}{lllll}
\operatorname{ERD}(\omega)_{1} & \operatorname{ERD}(\omega)_{2} & \ldots & \operatorname{ERD}(\omega)_{p} & \operatorname{ERD}(\omega)_{p+1}
\end{array}\right]^{T} .
\end{array}\right.
$$

The energy ratios in the erv $(\bar{\omega})_{d}$ do not change in comparison with the ones in theerv $(\bar{\omega})_{u}$, when the $\operatorname{erd}(\bar{\omega})=0$. Thus, the retaining wall is not damaged. The energy ratios in the erv $(\bar{\omega})_{d}$ fluctuate up and down based on the ones in the $\operatorname{erv}(\bar{\omega})_{u}$, when the $\operatorname{erd}(\bar{\omega}) \neq 0$. Thus, the wall is damaged. So, the damage state of the retaining wall can be detected qualitatively via the erd $(\bar{\omega})$. Similarly, the damage state of the retaining wall can also be detected qualitatively via the ERD $(\omega)$.

Based on the erd $(\bar{\omega})$ and ERD $(\omega)$, the damage identification indexes ersd (the energy ratio standard deviation) and ERSD (the Energy Ratio Standard Deviation) are defined as

$$
\left\{\begin{array}{l}
\operatorname{ersd}=\sqrt{\sum_{k=1}^{p+1}\left(\frac{\operatorname{erd}(\bar{\omega})_{k}}{p+1}\right)^{2}}, \\
\text { ERSD }=\sqrt{\sum_{k=1}^{p+1}\left(\frac{\operatorname{ERD}(\omega)_{k}}{p+1}\right)^{2}} .
\end{array}\right.
$$

The damage index ersd $=0$, when the $\operatorname{erd}(\bar{\omega})=0$. Thus, the retaining wall is not damaged. The damage index ersd $>0$, when the $\operatorname{erd}(\bar{\omega}) \neq 0$. Thus, the wall is damaged. The value of ersd becomes larger and larger, when the fluctuation intensity of erd $(\bar{\omega})$ becomes larger and larger. With the increase of the holes number, the values of the damage indexes become larger 
and larger. Thus, the either ersd or ERSD can be used to detect the damage state of the retaining wall quantitatively.

In a structural system, the variation of structural stiffness caused by damage will give rise to the variations of structural responses, which will cause the variations of IRF and VIRF. Similarly, ersd and ERSD, which are based on IRF and VIRF, respectively, also change. Especially, the stiffness in partial damage changes the most. Similarly, the values of ersd and ERSD in partial damage also change the most. Thus, the partial damage location can be detected by variation characteristics of ersd and ERSD.

In addition, the damage intensity of partial damage within walls can be identified by establishing a quantitative relationship between the damage intensity and identification index. The damage intensity can be calculated reversely via ersd or ERSD, if the quantitative relationship between the damage intensity and damage index is known. Consequently, the damage (the damage state, damage location, and damage intensity) of the retaining wall can be detected via ersd and ERSD.

2.4. Comparison of ERSD and ERSD. IRF is related to both the external excitation and structural responses. To calculate IRF, both the excitation and responses must be available. However, VIRF is only related to structural responses. VIRF can be calculated when virtual excitation and virtual response are available. To detect the damage state of the wall, the damage index ersd, which is based on IRF, requires that the external excitation and one structural response are available at least. The damage index ERSD, which is based on VIRF, requires that two structural responses are available at least. To diagnose the damage location, ersd requires $m$ measuring points, but ERSD requires $(m+1)$ points, where $m$ is the number of points. To acquire IRF and VIRF, the required sensors and signal transmission system are similar. The required excitation equipment for IRF must be the one that can record the excitation signal. In contrast, the excitation equipment for VIRF is just a common hammer. And, the excitation equipment for VIRF is not even required, when the excitations are such ambient ones as wind loads, vehicle loads. Thus, the equipment for ERSD are much simpler than the ones for ersd.

\section{Tests Analyses}

To identify the damage within the retaining wall via ersd and ERSD, vibration tests on a pile plate retaining wall (concrete wall) are performed, as shown in Figure 1. The geometrical parameters of the wall are listed in Table 2. Backfill behind the wall is sand, and soil in front of the wall is miscellaneous fill. Material parameters of the wall and backfill are shown in Table 3 .

As mentioned above, damages may occur and accumulate within the retaining wall due to many causes. In the light of literature $[29,30]$, the micro-cracks or holes are common damages in the structure. To simulate the actual damage within the retaining wall, holes are drilled in the wall, as shown in Figure 2. The parameters of the holes are listed in Table 4, where $L$ is the length of the wall and $\mathrm{H}$ is the height of the wall.

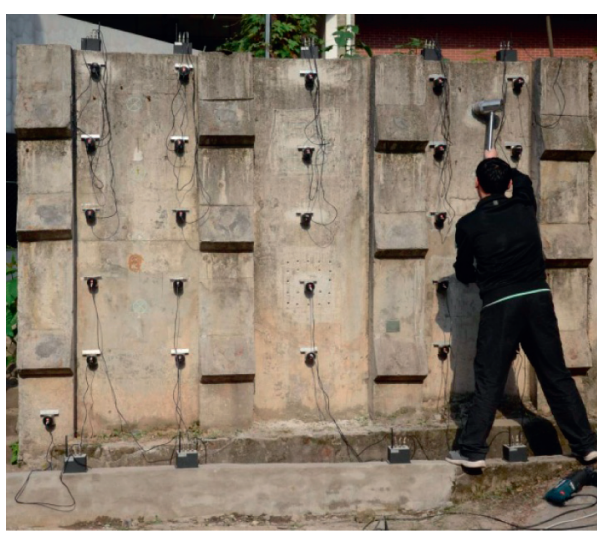

FIGURE 1: Vibration tests on the pile plate retaining wall.

TABLE 2: The geometrical parameters of the wall.

\begin{tabular}{lc}
\hline Geometrical parameters & Unit $(\mathrm{m})$ \\
\hline Height & 2.2 \\
Length & 3.0 \\
Thickness & 0.2 \\
Anchored depth & 0.6 \\
\hline
\end{tabular}

No holes are drilled in the wall, when the value of damage intensity is zero. With the increase of the number of holes, the value of damage intensity increases continuously. To describe the partial damage intensity, DI (Damage Intensity) is defined as

$$
\mathrm{DI}=\left(\frac{N \cdot V_{h}}{V}\right)
$$

where $N$ is the number of holes, $V_{h}$ is the volume of a single hole, and $V$ is the total volume of the partial area $(V=0.25 \mathrm{~m} \times 0.25 \mathrm{~m} \times 0.1 \mathrm{~m})$.

Generally speaking, equipment for model tests should include exciting hammers, sensors, and a signal transmission system. In this paper, the exciting hammer is a DFC-2 hammer, which can record hammer impulse signals automatically, as shown in Figure 3(a). The sensors are 941B accelerometers (The frequency range is $0.17-100 \mathrm{~Hz}$, the sensitivity is $0.3\left(\mathrm{~V} \cdot \mathrm{s}^{2} / \mathrm{m}\right)$. And, these sensors are fixed on the wall by binder, as shown in Figure 3(b). The signals recorded via accelerometers are collected by the signal collecting system, the JM3863A wireless vibration test system, as shown in Figure 3(c). The signals collected by the JM3863A are transmitted to a computer by the JM1802 gateway, as shown in Figure 3(d).

To localize the damage, 26 accelerometers are fixed on the wall, as shown in Figure 4. The IRF can be acquired via the excitation signal of DFC-2 hammer and response signals of point 1 to point 25 . In the light of literature [22, 23], the point with less response is selected as virtual excitation point, and other points are virtual responses points. Here, point 26 , whose response is much less, is selected as the reference point. And, points 1 to 25 are calculating points. The VIRF can be acquired via the virtual excitation and virtual responses. The locations of hammer excitation are the points from I to VI. The locations of measuring points and 
TABLE 3: Material parameters of the concrete and backfill.

\begin{tabular}{lccccc}
\hline Material & Density $\left(\mathrm{kg} / \mathrm{m}^{3}\right)$ & Elasticity modulus $(\mathrm{MPa})$ & Poisson's ratio & Cohesive force $(\mathrm{kPa})$ & Internal friction angle $\left(^{\circ}\right)$ \\
\hline Concrete & 2500 & $2.11 \times 104$ & 0.2 & - & - \\
Backfill behind the wall & 1901 & 15.4 & 0.3 & 0 & 37.6 \\
Backfill in front of the wall & 2385 & $2.3 \times 104$ & 0.2 & 460 & 39.5 \\
\hline
\end{tabular}

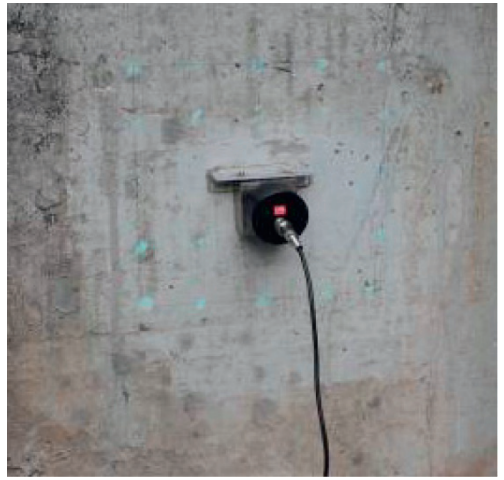

case 1 (no holes)

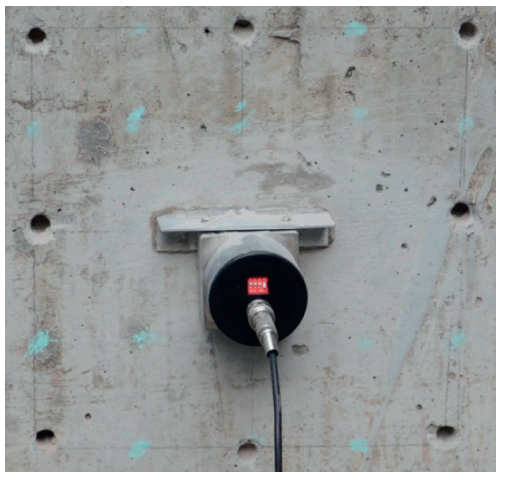

case 4 ( 8 holes)

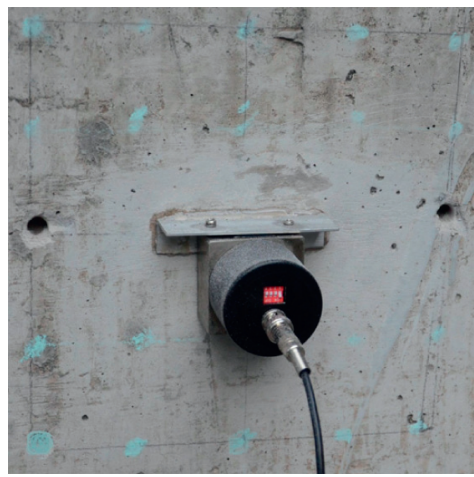

case 2 ( 2 holes)

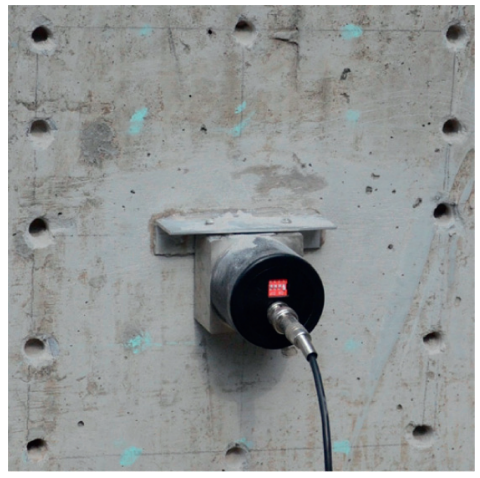

case 5 (12 holes)

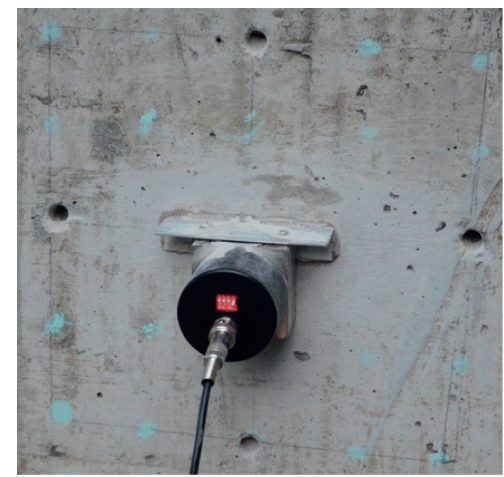

case 3 ( 4 holes)

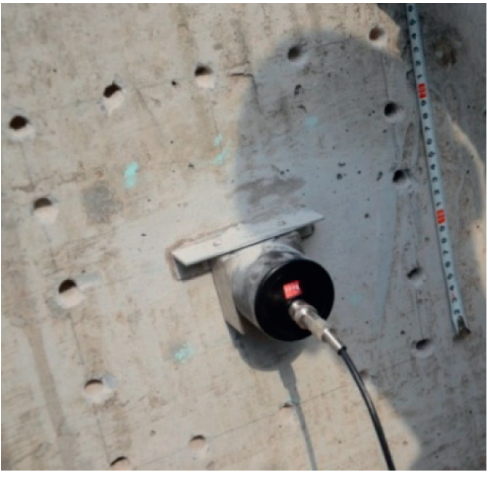

case 6 (16 holes)

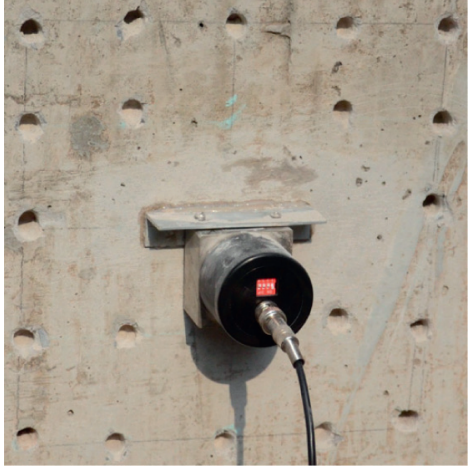

case 7 (20 holes)

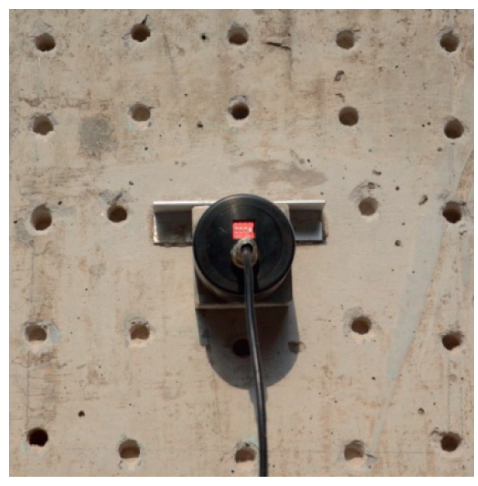

case 8 ( 24 holes $)$

Figure 2: Partial damages.

excitation points are shown in Figure 4. In the light of literature [29], under the effects of hammer excitation (low strain excitation), both the wall and filled soil vibrate mildly without considering the additional dynamic effects of the filled soil on the wall.

Here, eight damage cases are considered to identify the damage within the retaining wall, as listed in Table 5. Case 1 is the undamaged case, and cases $2-8$ are damaged cases. The test steps are listed here. (1) The vibration tests on the wall should be performed three times at each excitation point under every damage case to reduce the error. (2) The response signals and excitation ones are collected and transmitted to a computer. (3) The IRF and VIRF are acquired via data processing. (4) The Hilbert marginal energy 
TABLE 4: The parameters of the holes.

\begin{tabular}{lc}
\hline Geometrical parameters & Unit \\
\hline Diameter of a single hole & $0.2 \mathrm{~m}$ \\
Depth of a single hole & $0.1 \mathrm{~m}$ \\
Area of holes drilled & $0.25 \mathrm{~m} \times 0.25 \mathrm{~m}=6.25 e^{-2} \mathrm{~m}^{2}$ \\
Scope of holes drilled & $\{\mathrm{L} \in(1.375 \mathrm{~m}, 1.625 \mathrm{~m}), \mathrm{H} \in(0.875 \mathrm{~m}, 1.125 \mathrm{~m})\}$ \\
\hline
\end{tabular}

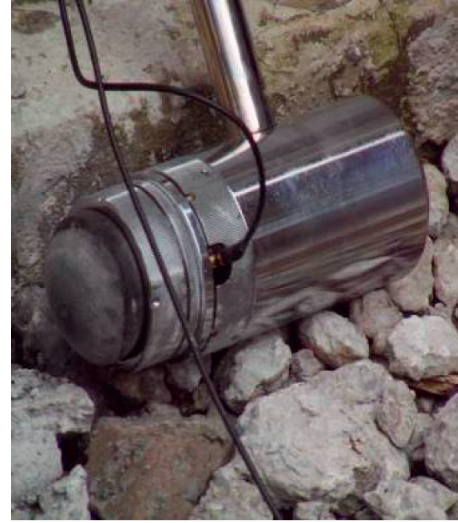

(a)

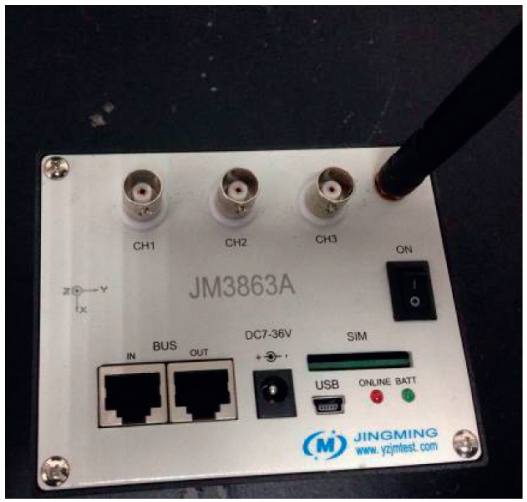

(c)

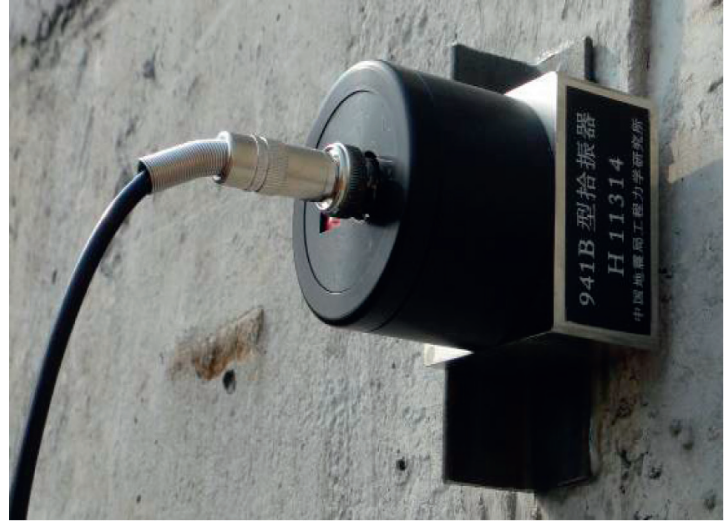

(b)

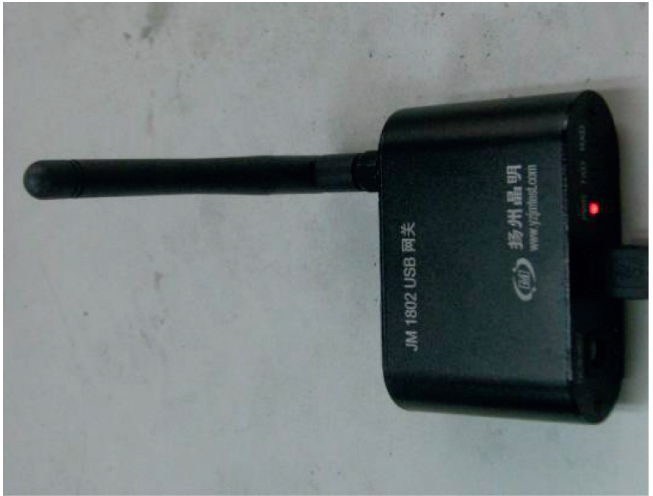

(d)

FIgURE 3: Exciting equipment, sensors, signal collecting system, and signal transmission system of vibration tests. (a) DFC-2 exciting hammer. (b) 941B sensor. (c) JM3863A vibration test system. (d) JM1802 wireless gateway.

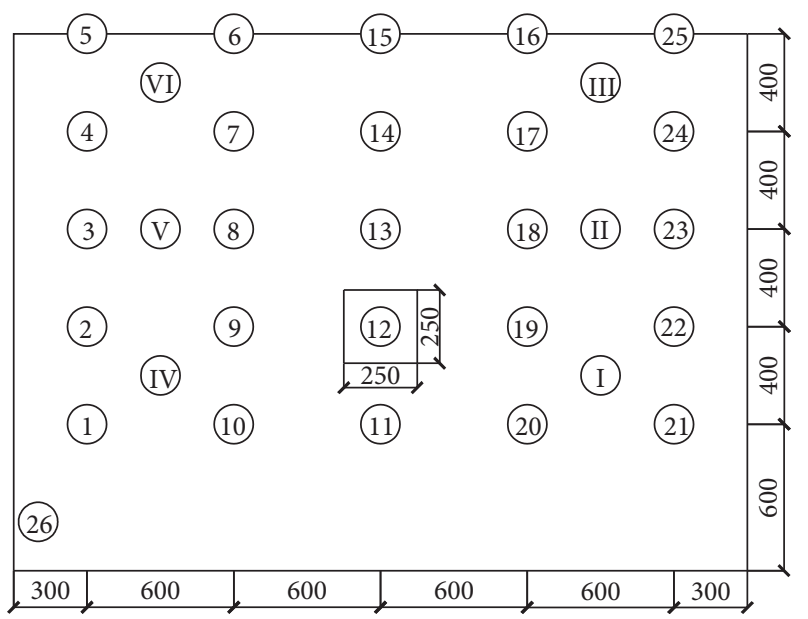

FIGURE 4: Assignment of sensors and excitation. 
Table 5: Damage cases.

\begin{tabular}{lcccccccc}
\hline Case & Case 1 & Case 2 (\%) & Case 3 (\%) & Case 4 (\%) & Case 5 (\%) & Case 6 (\%) & Case 7 (\%) & Case 8 (\%) \\
\hline DI & 0 & 1.00 & 2.00 & 4.02 & 6.03 & 8.04 & 10.05 & 12.06 \\
\hline
\end{tabular}

ratio spectrums are obtained via the Hilbert-Huang Transform of the IRF and VIRF. (5) The feature bands are selected via analyzing the damage sensitivity of bands. (6) The Hilbert feature vector and Hilbert damage feature vector are created, and the ersd and ERSD are calculated. (7) The damage state of the wall is detected. (8) The damage location of the wall is diagnosed. (9) The damage intensity of the wall is identified.

3.1. Detecting the Damage State of the Wall. Based on the signal processing technology, IRF and VIRF are obtained. The time of IRF curves is very short, because the duration of hammer excitation is about $0.001 \mathrm{~s}$. Thus, the IRF curves within $0.06 \mathrm{~s}$ are extracted to identify damage, as shown in Figure 5. And, the effective duration of VIRF curves is within $6 \mathrm{~s}$ after signal processing, as shown in Figure 5. Obviously, the time history curves of IRF nearly overlap under different damage cases. Similarly, the curves of VIRF also nearly overlap. Thus, it is difficult to extract damage information via the IRF curves and VIRF ones.

To detect the damage state of the wall, the Hilbert-Huang Transforms of IRF and VIRF are done. In the light of literature [31], the optimum value of EMD order is less than ten, so the value of EMD (the Empirical Mode Decomposition) order is ten. Thus, ten intrinsic mode functions can be acquired via EMDs of IRF and VIRF, respectively. And, ten energy ratios of sub-bands can be obtained via HT (the Hilbert Transform) of the intrinsic mode functions. The Hilbert marginal energy ratio spectrums of IRF and VIRF are shown in Figure 6. Due to the influence of the retaining wall with lower frequencies, the majority of sub-bands' energies are distributed over the minority of bands with lower frequencies. The total energy of the first five bands with lower frequencies is almost more than $90 \%$ of total energy, as shown in Figure 6.

The energy ratio of the same band changes, when retaining wall changes from an undamaged state to damaged one. The energy ratio variations caused by damage are nearly undetectable in such bands with higher frequencies as $7^{\text {th }}$ band to $10^{\text {th }}$ one. Thus, it is unnecessary to use all of the bands to identify damage. Only these bands, which are more sensitive to damage, are more useful for identifying damage, such as $1^{\text {st }}$ band to $6^{\text {th }}$ one of IRF and $1^{\text {st }}$ band to $5^{\text {th }}$ one of VIRF are much more sensitive to damage. In contrast, $7^{\text {th }}$ band to $10^{\text {th }}$ one of IRF and $6^{\text {th }}$ band to $10^{\text {th }}$ one of VIRF are not sensitive to damage, as is shown in Figure 6 (point 12). These bands, which are not sensitive to damage, are merged into the residual band. The values of the residual bands are listed in Table 6. Obviously, the values of the majority of residual bands are small. Nevertheless, the values of residual bands of IRF to point 1 are much larger; this is caused by the larger threshold value $\varepsilon_{0}$. The number of feature bands is determined by threshold value $\varepsilon_{0}$. The number of bands will become small, if the value of $\varepsilon_{0}$ is big. On the contrary, the number of bands will become large, if the value of $\varepsilon_{0}$ is small. Some bands, which are sensitive to damage, may be merged into the residual band due to the large $\varepsilon_{0}$. This may result in damage information being missed. On the contrary, some bands, which are much less sensitive to damage, may be added into feature bands due to small $\varepsilon_{0}$. This may decrease the effect of feature bands on damage identification. Because the damage sensitivities to different points are different, the value of $\varepsilon_{0}$ is variable for different points, as shown in Figure 7. Generally speaking, the values of $\varepsilon_{0}$ should be smaller for those points whose damage sensitivities are much poorer. And, the values of $\varepsilon_{0}$ should be larger for those points whose damage sensitivities are much stronger. Thus, the threshold value $\varepsilon_{0}$ plays a prominent role in extracting feature bands.

Then, the Hilbert feature vector, which is sensitive to damage, is created via the threshold $\varepsilon_{0}$ to replace the Hilbert marginal energy ratio spectrum, as shown in Figure 7. Although the value of the band energy is the largest, the damage sensitivity of this band is not necessarily the strongest, as shown in Figure 7 (the energy of $3^{\text {rd }}$ band is the largest, but its damage sensitivity is not the strongest, as shown in Figures 7(c) and 7(d)).

Based on the Hilbert feature vector, the Hilbert damage feature vector is created, as shown in Figure 8. As mentioned above, the wall is undamaged when damage feature vector is a zero vector. The wall is damaged when damage feature vector is a nonzero vector. Obviously, all the damage feature vectors are nonzero vectors, when holes are drilled in the wall. Thus, the damage state of the pile plate retaining wall can be sensitively distinguished via damage feature vectors which are based on IRF or VIRF.

Under the influences of the excitation location and damage location, the damage sensitivities of different measuring points are different. For instance, the damage sensitivity of point 7 which is far away from the partial damage is much poorer, while the one of point 12 which is located in damage location is much stronger.

3.2. Diagnosing the Damage Location of the Wall. As mentioned above, the damage indexes which are based on IRF or VIRF changes a lot in the location of partial damage. Thus, the damage location of the wall can be diagnosed via variation characteristics of the damage index. In the light of (21), ersd and ERSD are calculated under the different damage cases, as listed in Table 7. Firstly, the damage index values of these 25 measuring points are calculated under different damage cases. Then, a damage index trend surface can be formed by these 25 damage index values via MATLAB program under a certain damage case. The damage location of the wall can be diagnosed via the variation properties of the trend surface, as shown in Figure 9. 
TABLE 6: The energy ratios of the residual bands (the second time test at excitation point I).

\begin{tabular}{|c|c|c|c|c|c|}
\hline \multicolumn{2}{|c|}{ Ponits } & Case 1 & Case 3 & Case 6 & Case 8 \\
\hline \multirow{2}{*}{ Point 1} & IRF & 27.6610 & 27.8356 & 27.9351 & 27.9515 \\
\hline & VIRF & 5.1390 & 5.1219 & 5.1226 & 5.1277 \\
\hline \multirow{2}{*}{ Point 7} & IRF & 2.2895 & 2.2730 & 2.2615 & 2.2583 \\
\hline & VIRF & 3.9652 & 4.1001 & 4.1241 & 4.1184 \\
\hline \multirow{2}{*}{ Point 12} & IRF & 2.1113 & 2.8389 & 3.6641 & 4.5274 \\
\hline & VIRF & 5.2977 & 5.4774 & 5.6092 & 5.7922 \\
\hline \multirow{2}{*}{ Point 25} & IRF & 5.4611 & 5.3479 & 5.3195 & 5.3163 \\
\hline & VIRF & 5.6343 & 5.7138 & 5.6542 & 5.6128 \\
\hline
\end{tabular}

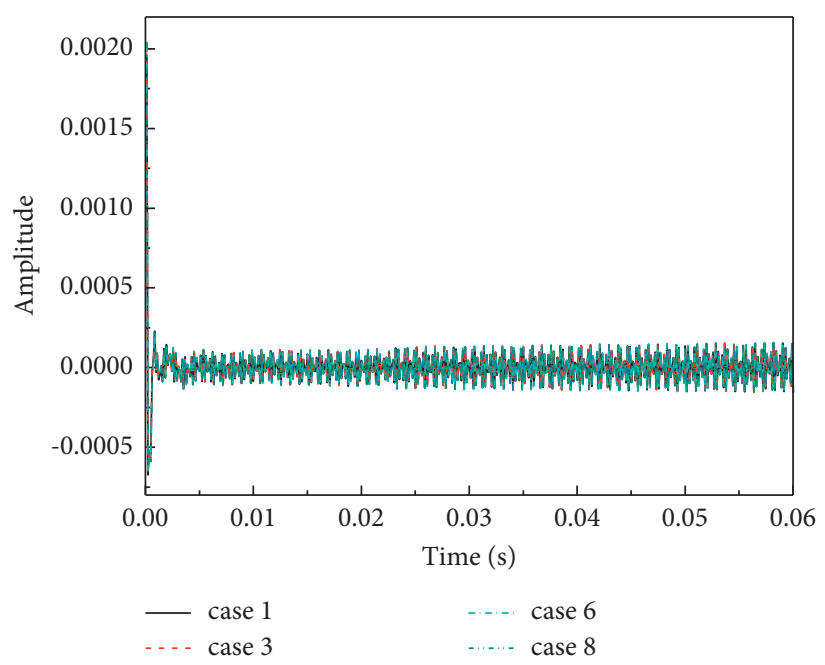

(a)

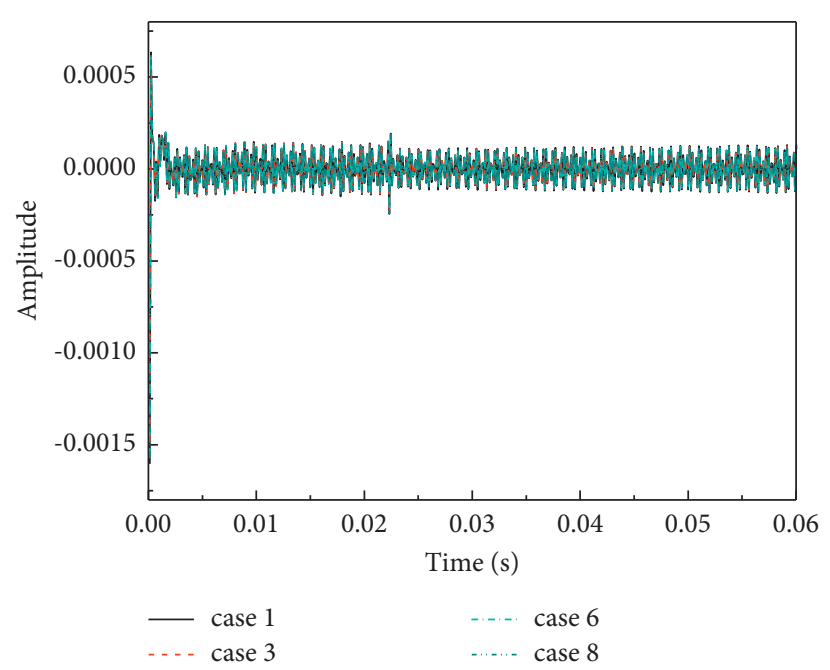

(c)

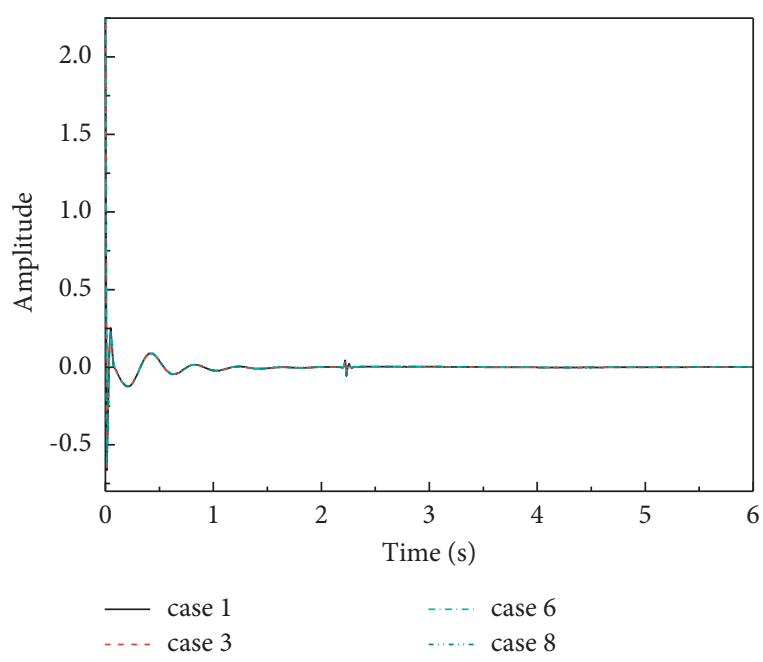

(b)

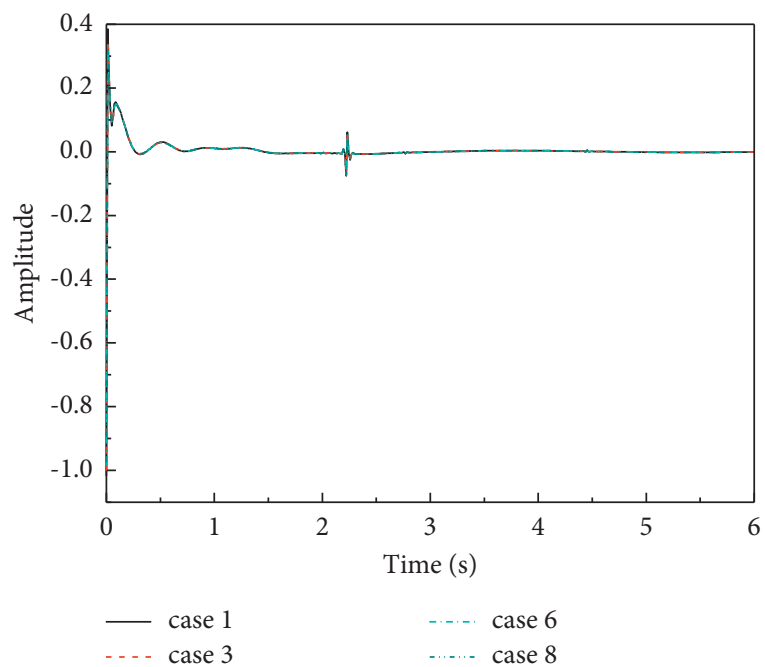

(d)

Figure 5: Continued. 


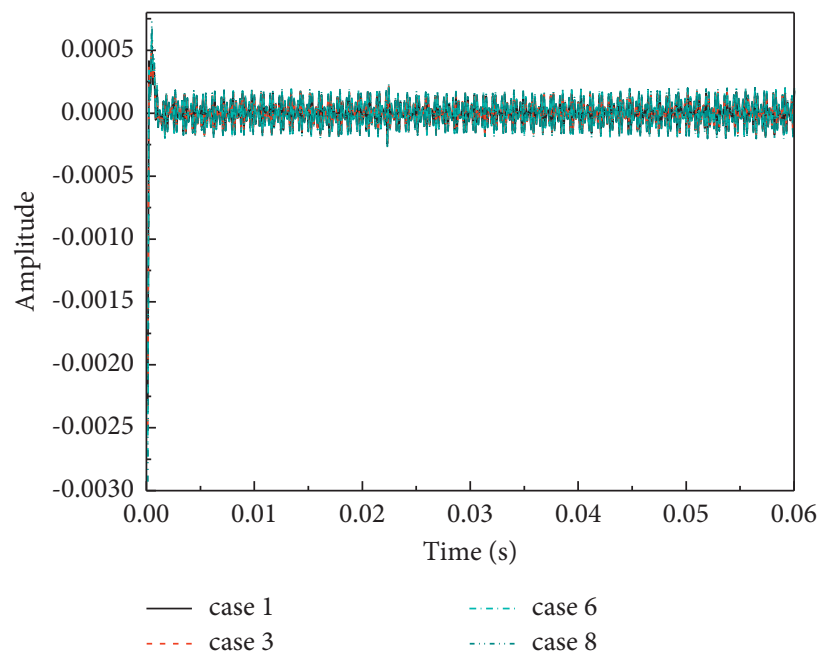

(e)

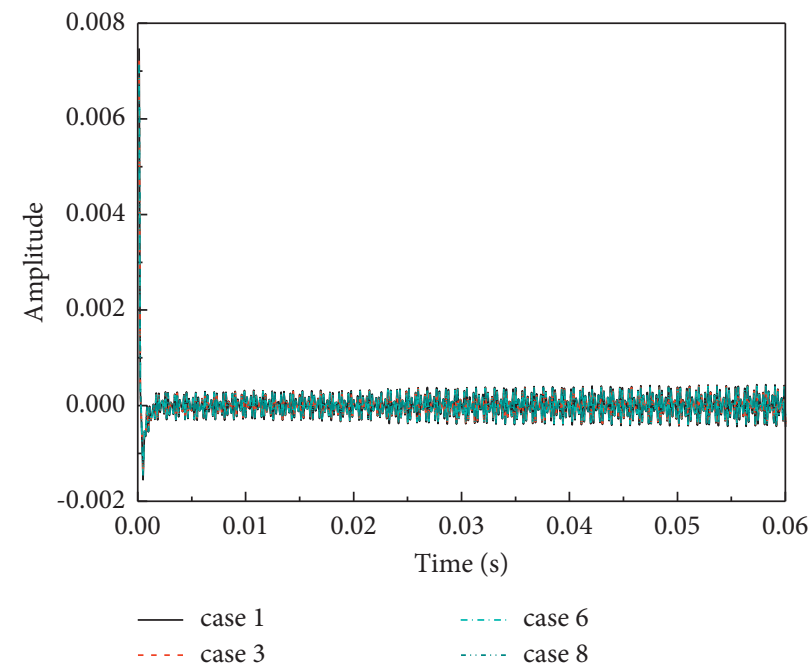

(g)

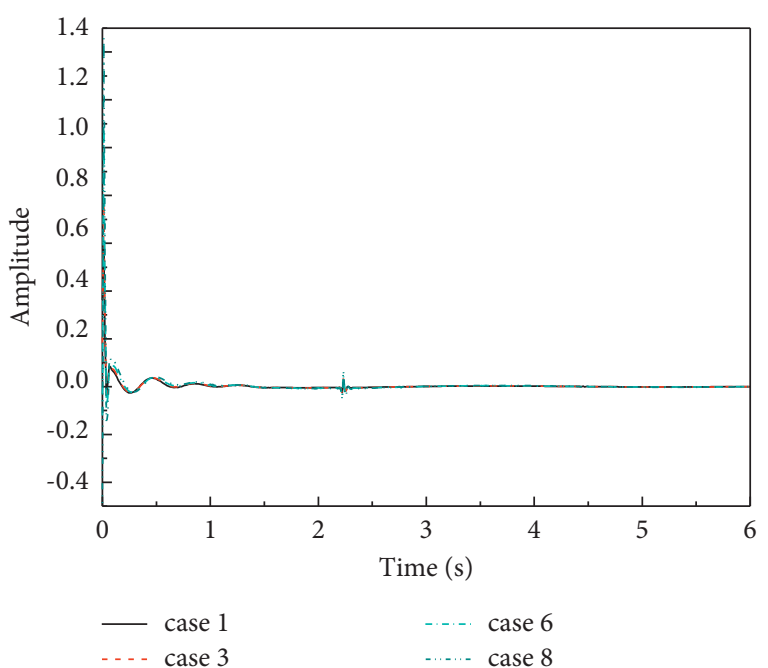

(f)

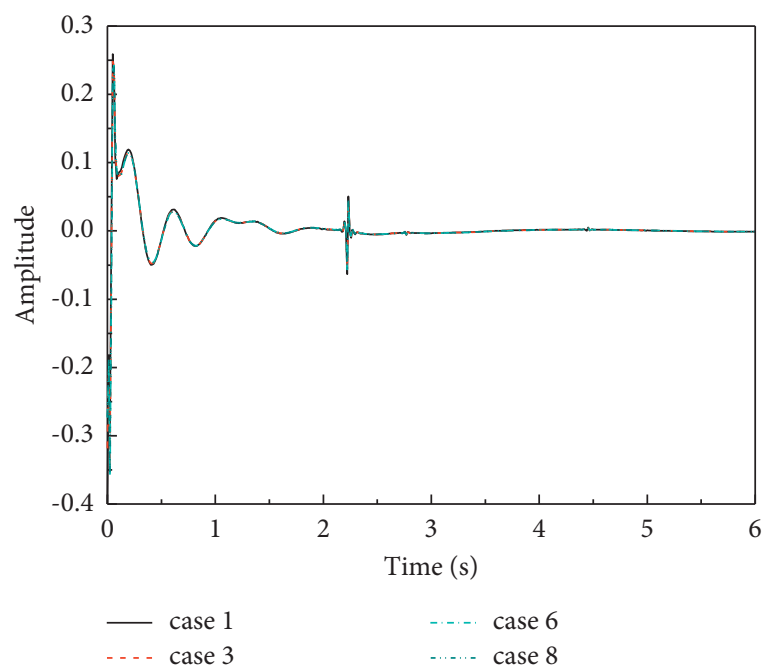

(h)

FIgURE 5: The time history curves of IRF and VIRF of different measuring points (the second time test at excitation point I). Point 1: (a) IRF. (b) VIRF. Point 7: (c) IRF. (d) VIRF. Point 12: (e) IRF. (f) VIRF. Point 25: (g) IRF. (h) VIRF.

Obviously, the peak coordinate of the trend surface is located in the center of the scope $\{L \in(1.375 \mathrm{~m}, 1.625 \mathrm{~m})$, $H \in(0.875 \mathrm{~m}, 1.125 \mathrm{~m})\}$, where the partial damage is simulated. Thus, the damage location of the wall can be diagnosed via the peak coordinate of the damage index trend surface. In comparison with ersd, the peak characteristics of ERSD trend surface caused by partial damage are much better than the ones of ersd trend surface. Especially, the damage location can be detected more effectively and accurately via ERSD trend surface, when DI is small (Figure 9(a)). What calls for special attention is that the values of the damage indexes are nonnegative. The negative values in trend surface are caused by the B-spline technique.

3.3. Identifying the Damage Intensity of the Wall. As mentioned above, DI can be calculated reversely via the damage index, when the quantitative relationship between the damage intensity and damage index is known. Here, $\mathrm{DI}$ is the one of partial damages (measuring point 12) that is diagnosed via the damage index trend surface. To establish the quantitative relationship between the damage intensity and damage index, the quantitative relationship between damage index and DI of point 12 is fitted by polynomial fitting. Firstly, the mean value of the damage index of point 12 is calculated under the same damage intensity at different exciting locations. Then, the quantitative relationship between the damage index and damage intensity of point 12 can be acquired. The polynomial coefficient will become much larger, if the relationship between the damage index and DI which is much less than the value of damage index is fitted directly. Thus, the quantitative relationship between di (the relative damage intensity) and damage index is fitted. Finally, the quantitative relationship between damage index and di $(\mathrm{di}=\mathrm{DI} \times 100)$ can be expressed as: 

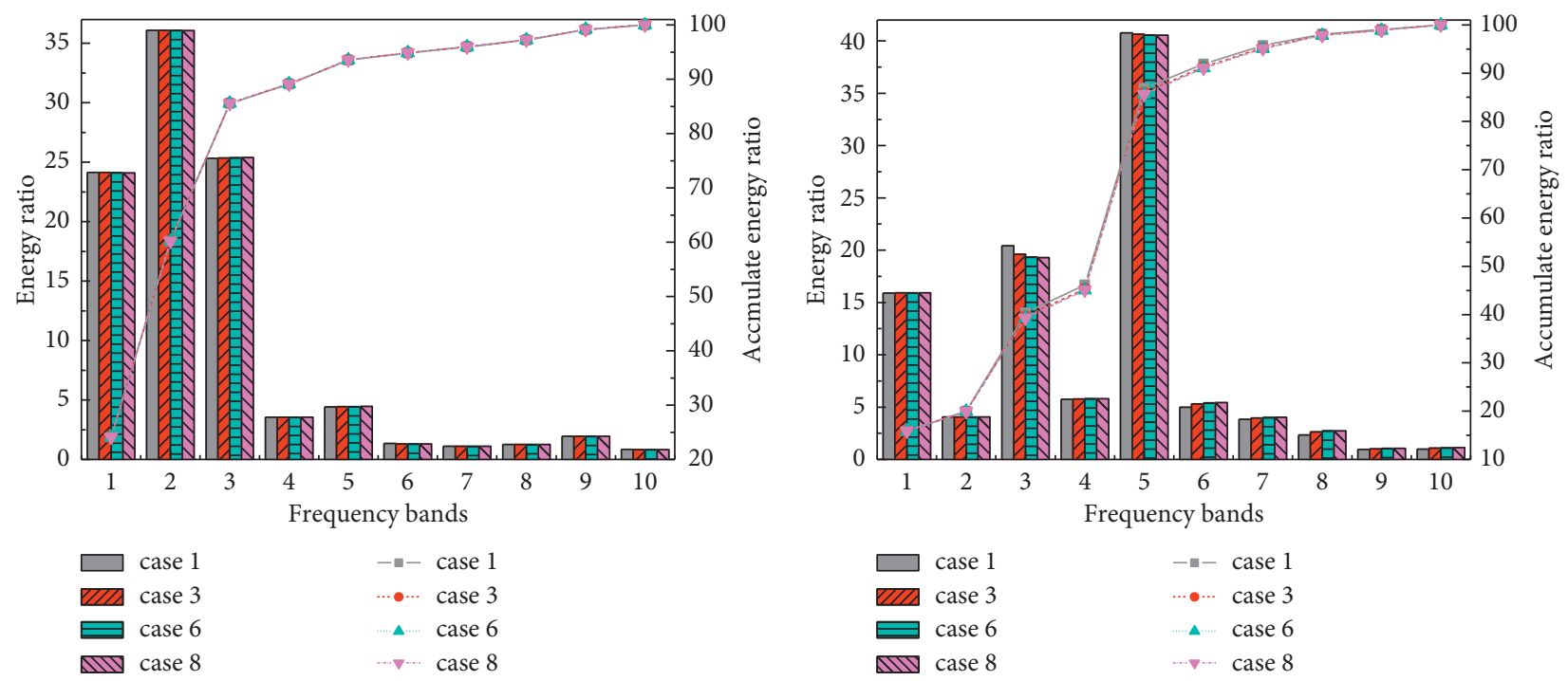

(a)
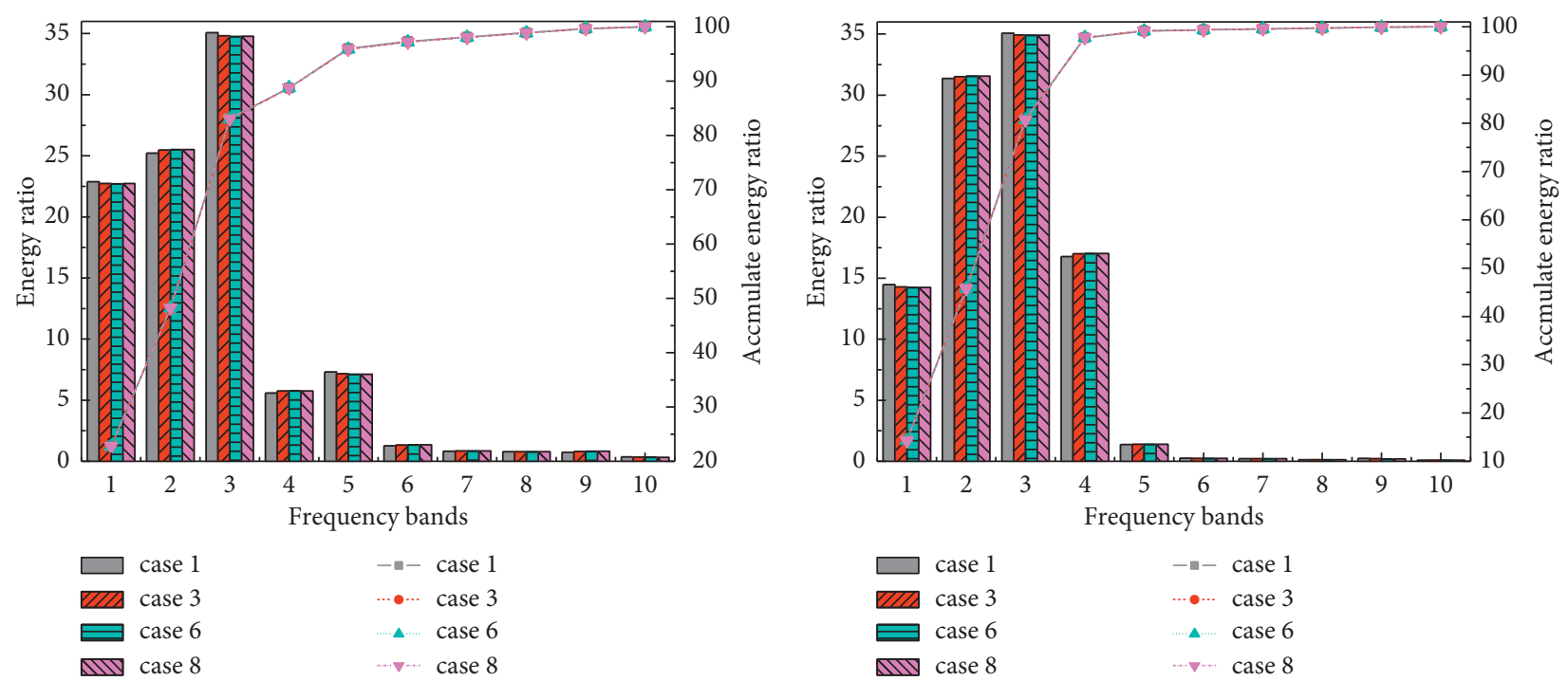

(c)
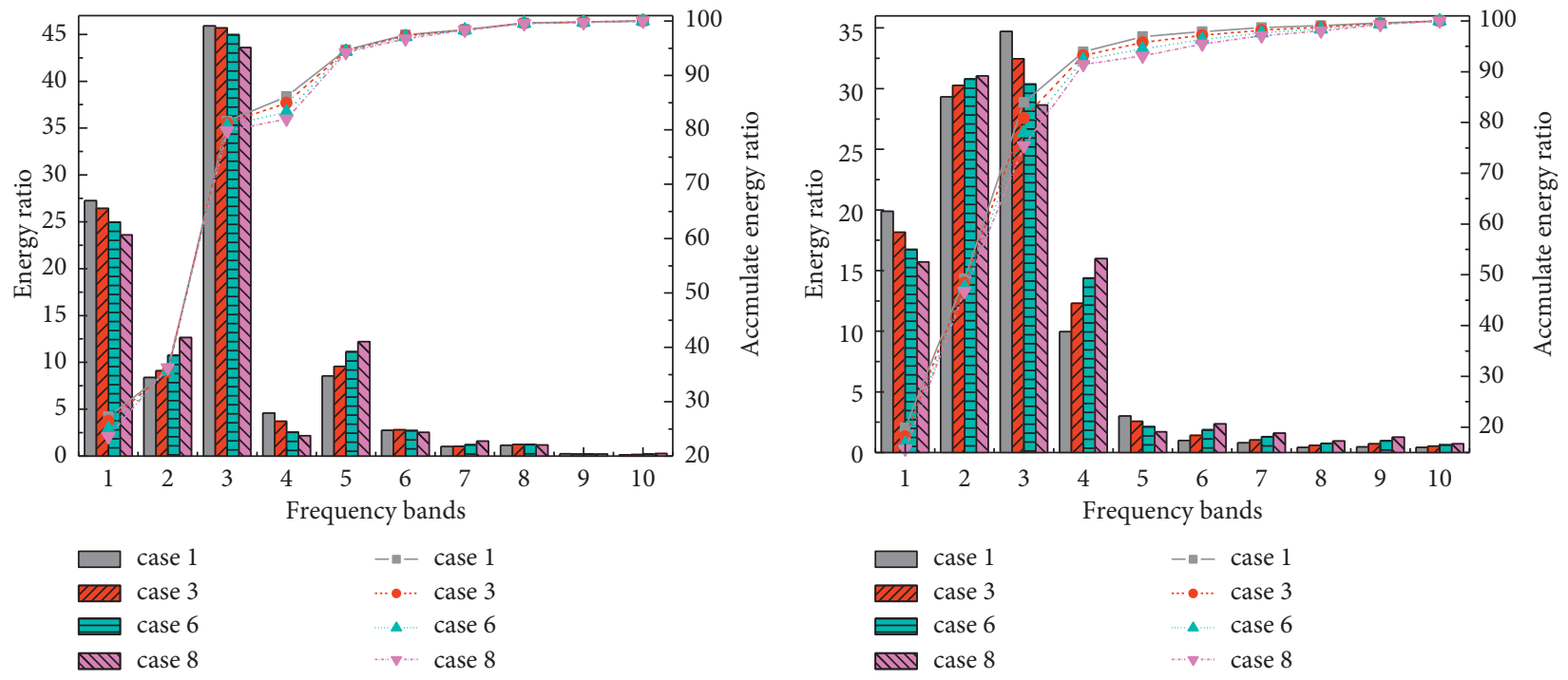

(e)

(f)

Figure 6: Continued. 


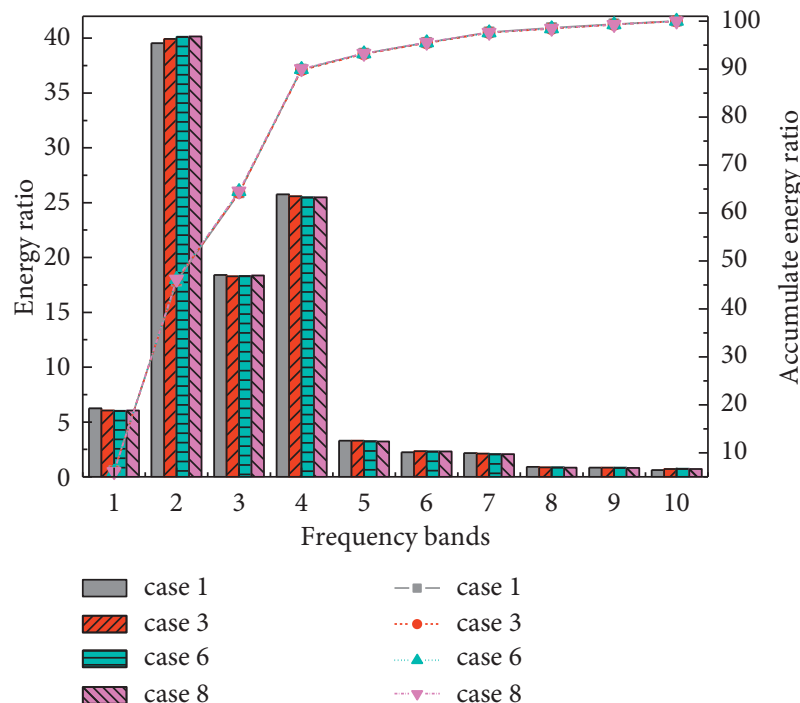

(g)

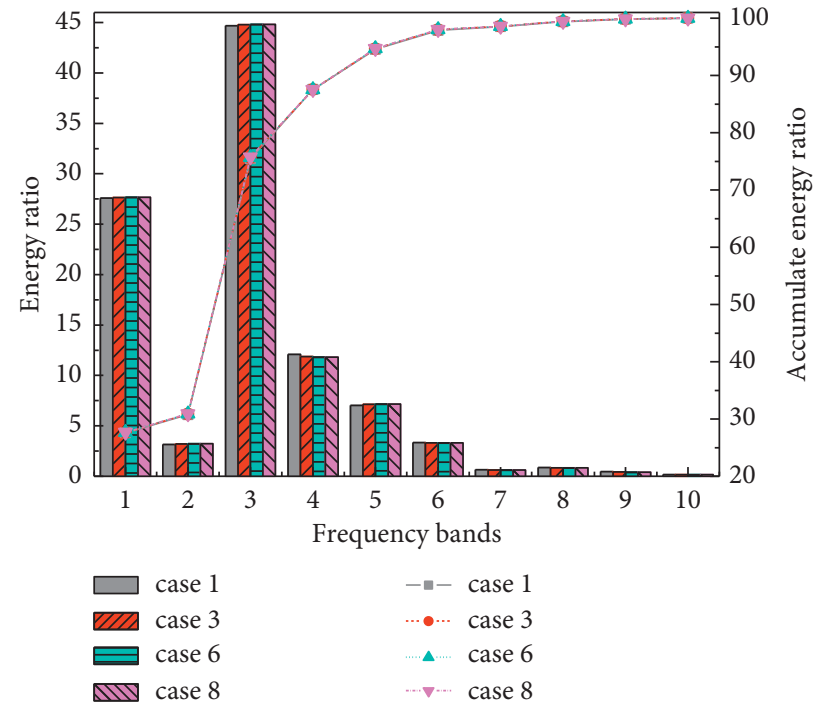

(h)

FIgURE 6: The Hilbert marginal energy ratio spectrum of IRF and VIRF (the second time test at excitation point I). Point 1: (a) IRF. (b) VIRF. Point 7: (c) IRF. (d) VIRF. Point 12: (e) IRF. (f) VIRF. Point 25: (g) IRF. (h) VIRF.

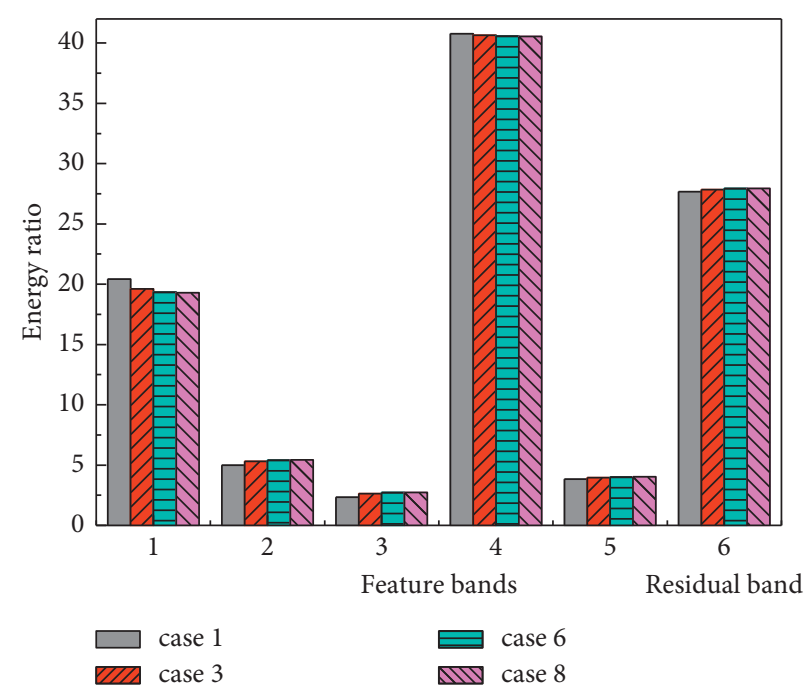

(a)

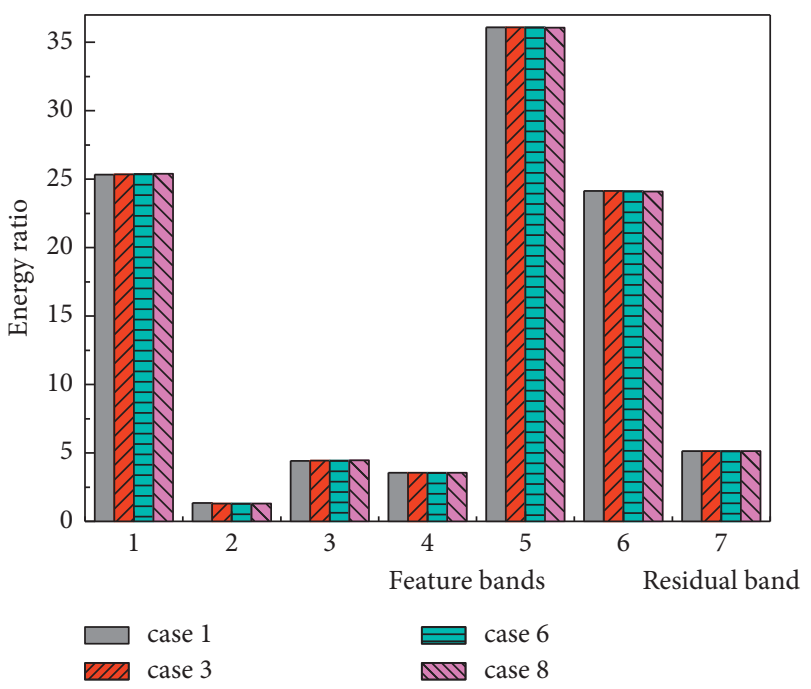

(b)

FIgURE 7: Continued. 

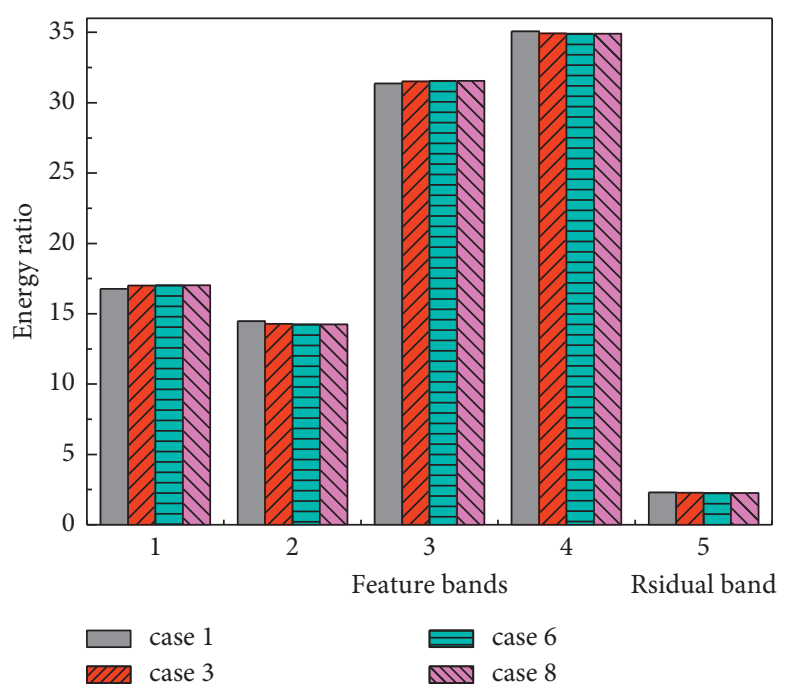

(c)

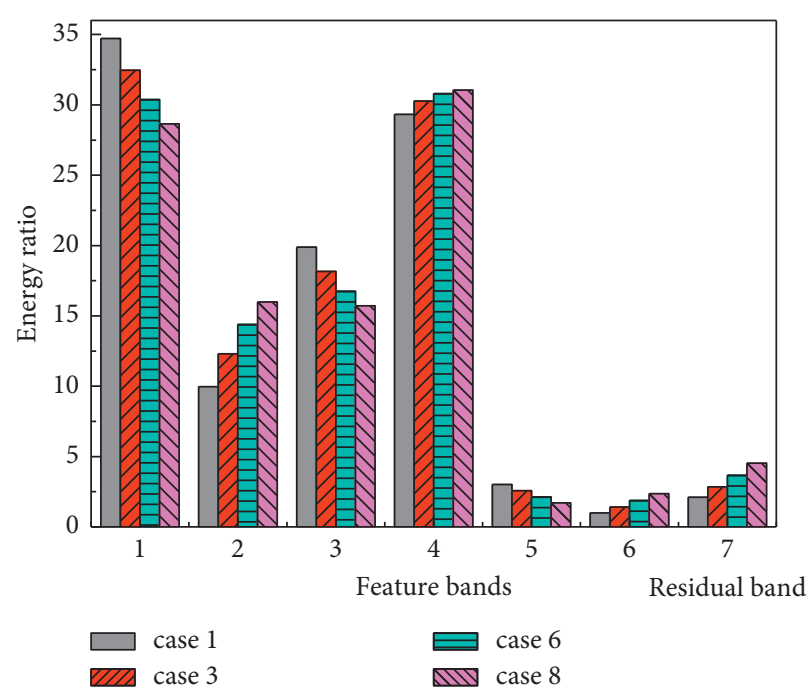

(e)

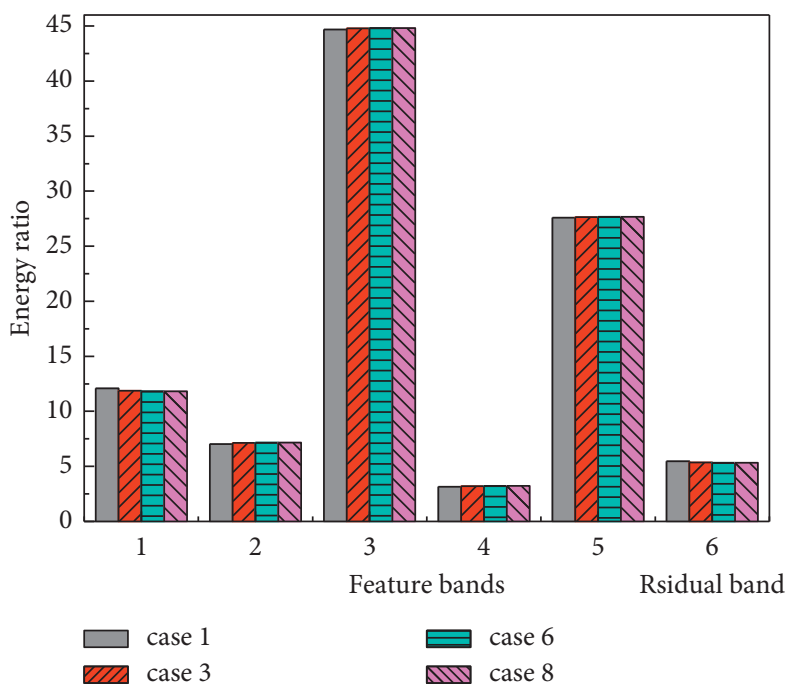

(g)

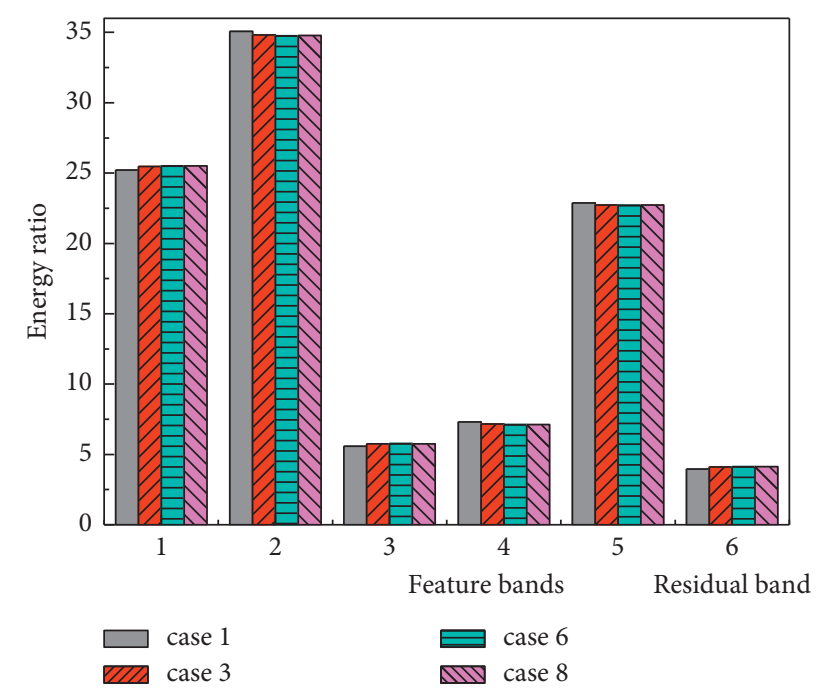

(d)

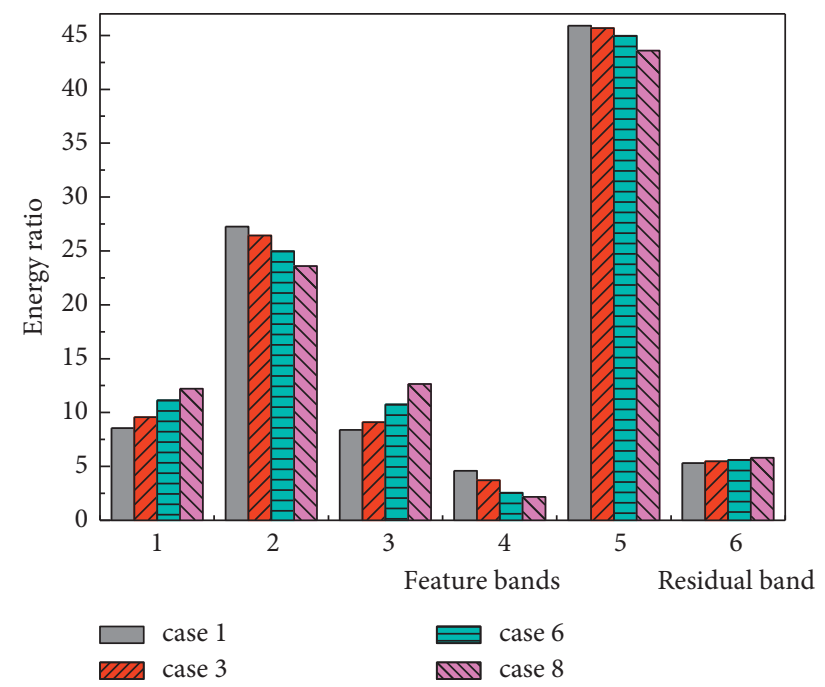

(f)

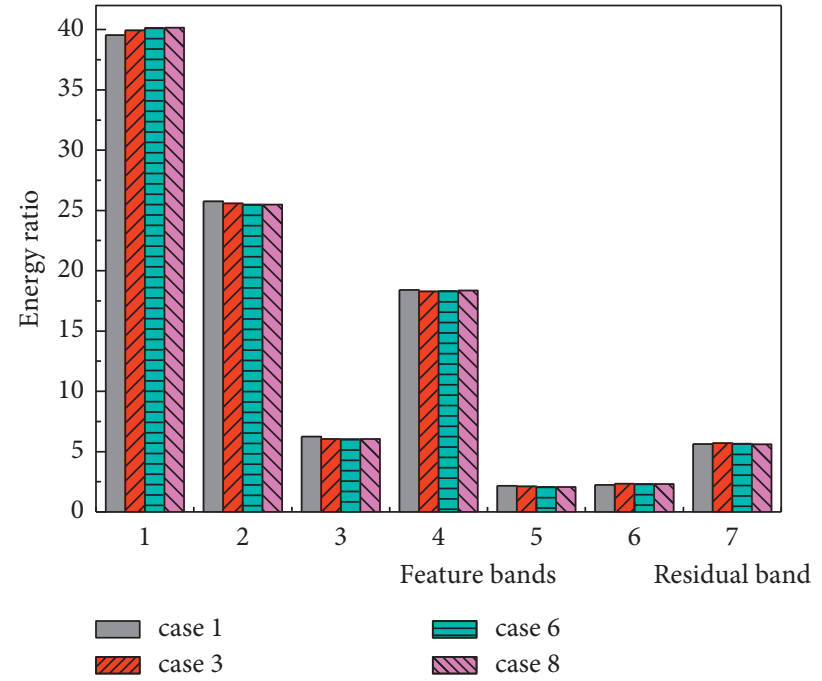

(h)

FIGURE 7: The Hilbert feature vector of IRF and VIRF (the second time test at excitation point I). Point 1: (a) IRF $\left(\varepsilon_{0}=0.2\right)$. (b) VIRF $\left(\varepsilon_{0}=0.25\right)$. Point 7: (c) IRF $\left(\varepsilon_{0}=0.15\right)$. (d) VIRF $\left(\varepsilon_{0}=0.25\right)$. Point 12: (e) IRF $\left(\varepsilon_{0}=0.15\right)$. (f) VIRF $\left(\varepsilon_{0}=0.2\right)$. Point 25: (g) IRF $\left(\varepsilon_{0}=0.25\right)$. (h) $\operatorname{VIRF}\left(\varepsilon_{0}=0.15\right)$. 


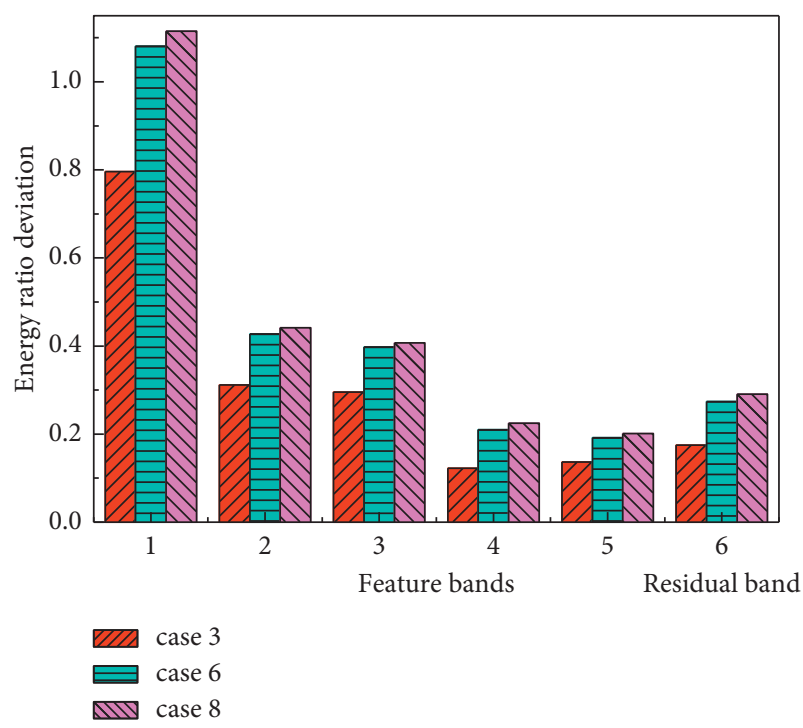

(a)

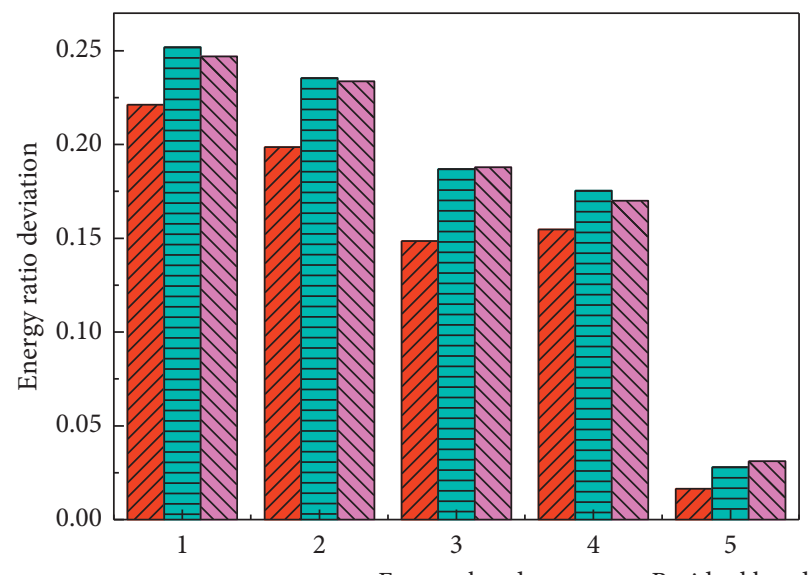

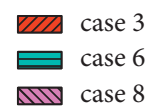

(c)

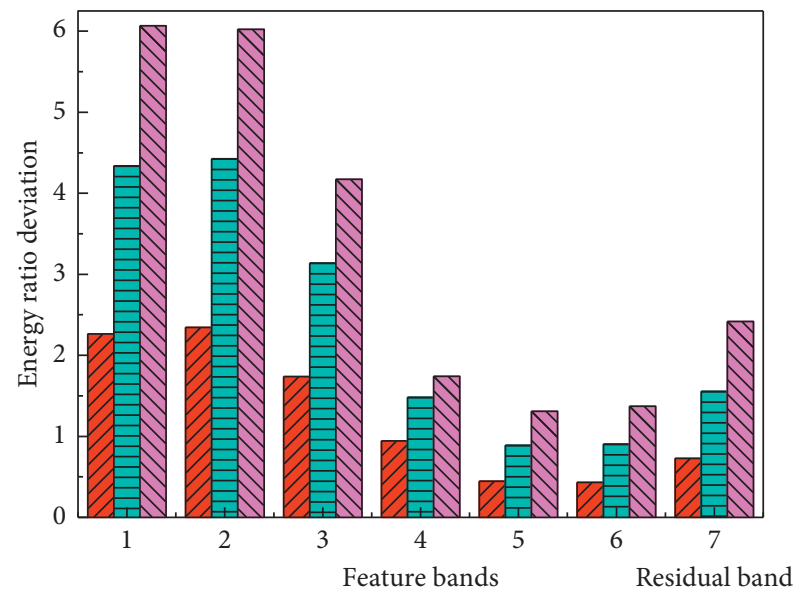

IIn case 3

ए case 6

חा case 8

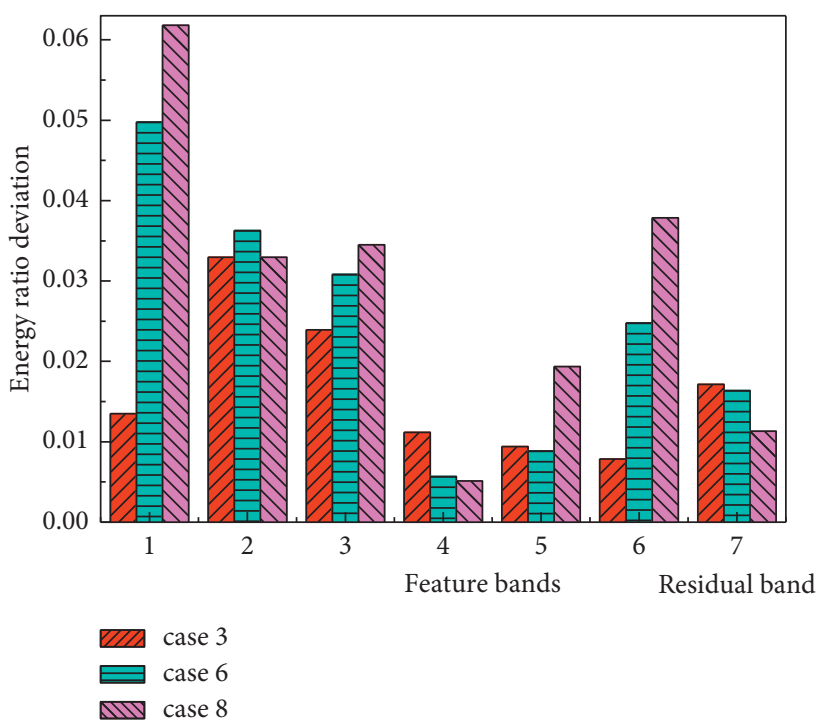

(b)

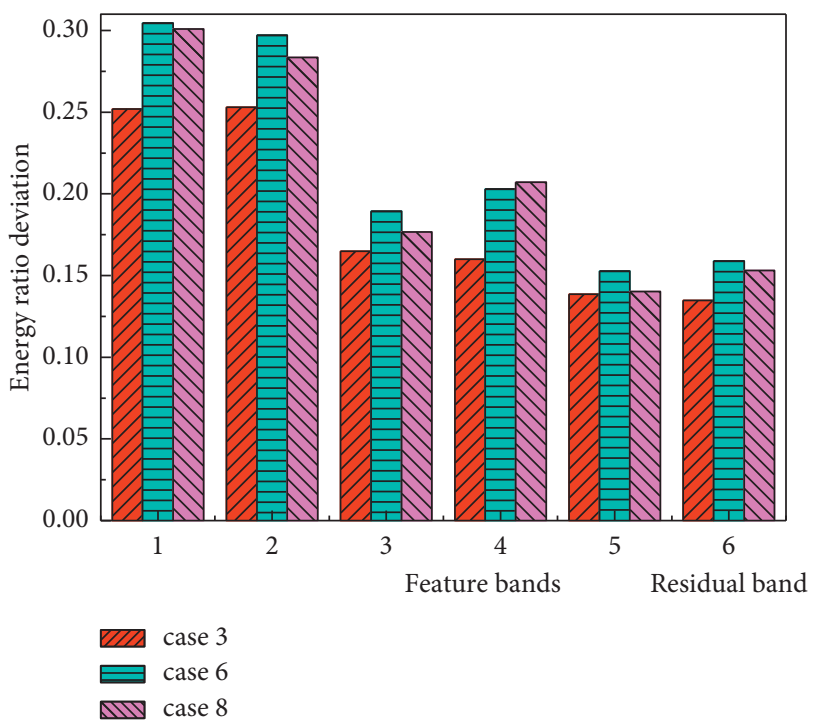

(d)

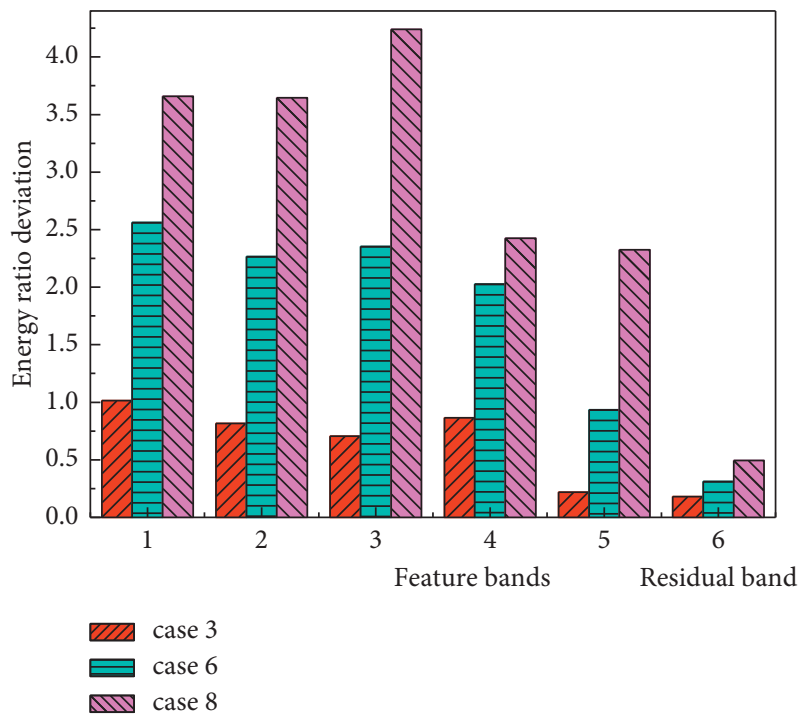

(f)

Figure 8: Continued. 


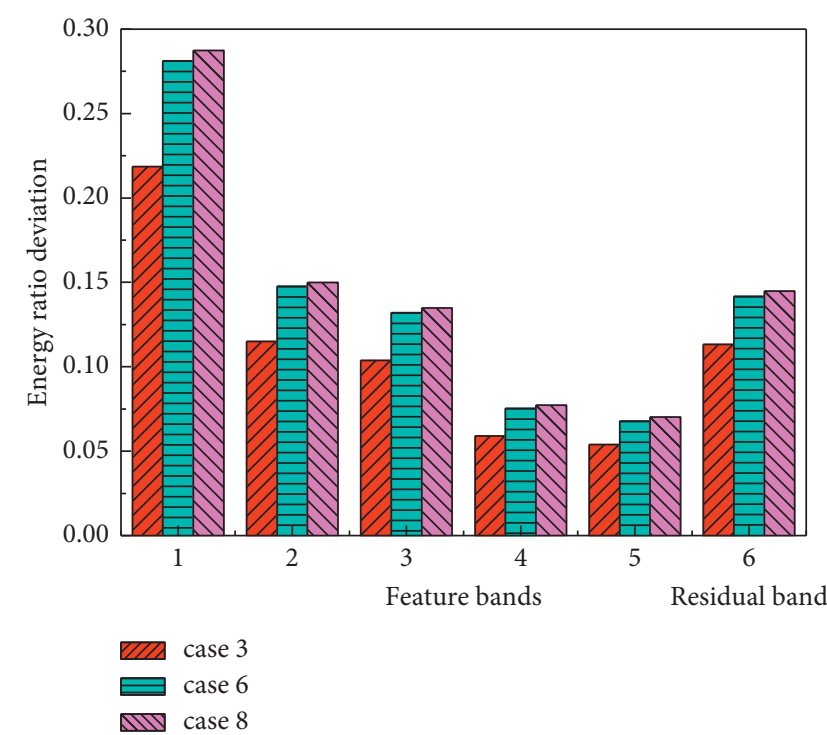

(g)

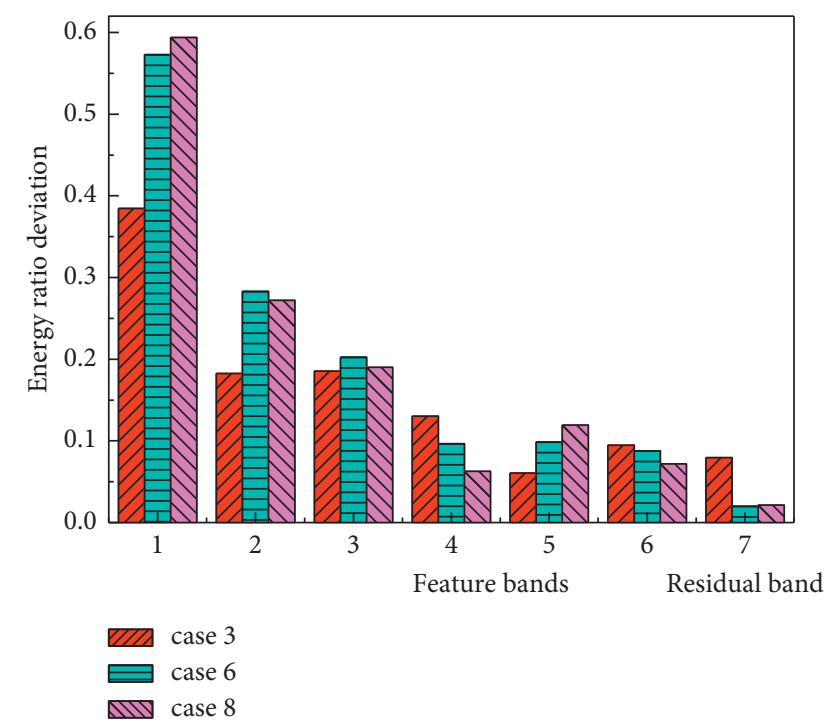

(h)

Figure 8: The Hilbert damage feature vector of IRF and VIRF (the second time test at excitation point I). Point 1: (a) IRF. (b) VIRF. Point 7: (c) IRF. (d)VIRF. Point 12: (e) IRF. (f) VIRF. Point 25: (g) IRF. (h) VIRF.
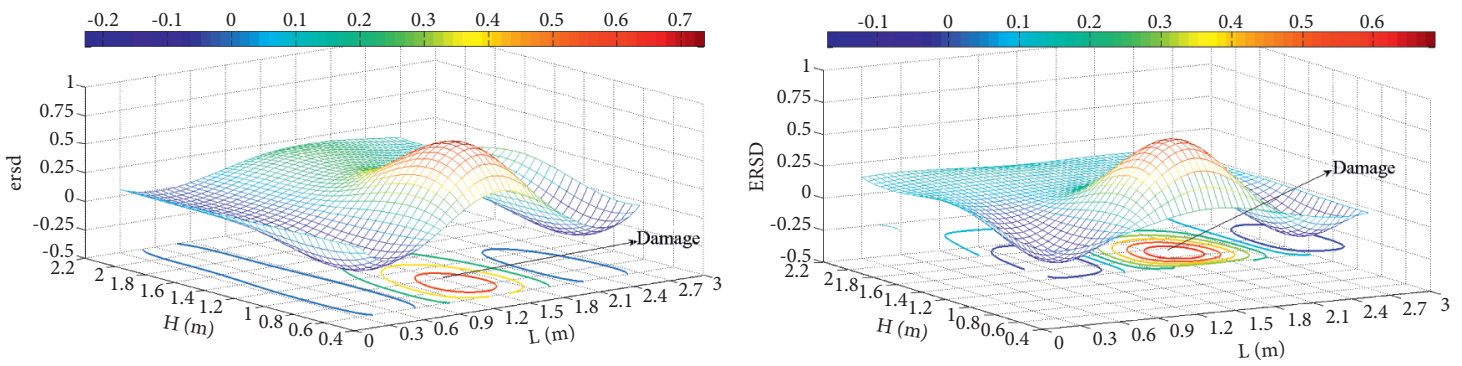

(a)
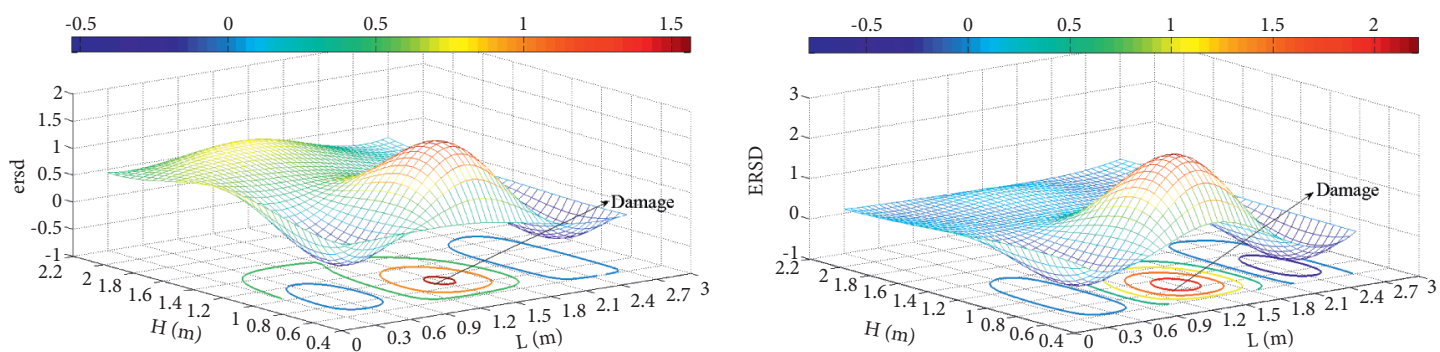

(b)
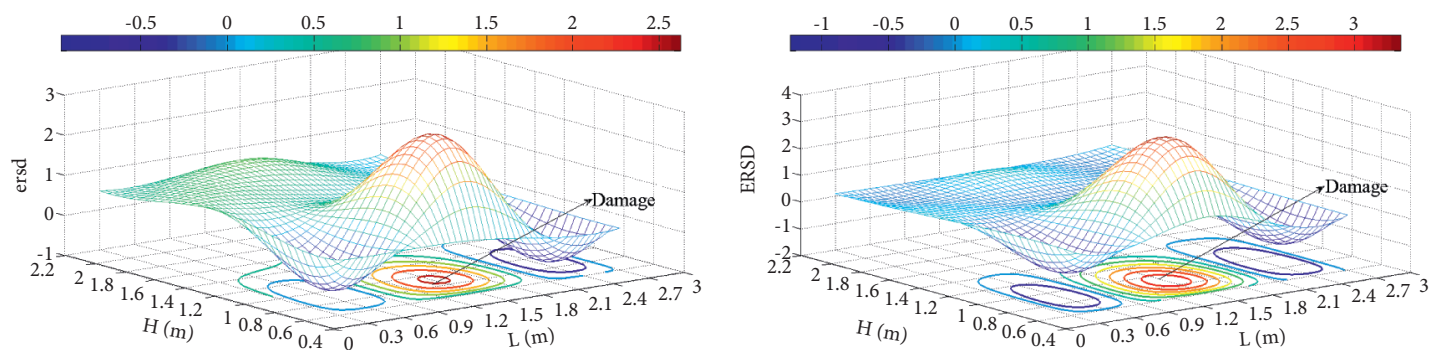

(c)

Figure 9: Trend surface of the damage index (the second time test at excitation point I). (a)Case 3. (b) Case 6. (c) Case 8. 
TABLE 7: The values of damage indexes of different points (the second time test at excitation point I).

\begin{tabular}{lcccrr}
\hline Points & Damage index & Case 1 & Case 3 & Case 6 & Case 8 \\
\hline \multirow{2}{*}{ Point 1} & ersd & 0 & 0.0302 & 0.0466 & 0.0529 \\
& ERSD & 0 & 0.0216 & 0.0309 & 0.0352 \\
\hline \multirow{2}{*}{ Point 7} & ersd & 0 & 0.0224 & 0.0245 & 0.0343 \\
& ERSD & 0 & 0.0309 & 1.5044 & 1.8320 \\
\multirow{2}{*}{ Point 12} & ersd & 0 & 0.5582 & 0.1447 & 0.0457 \\
\hline \multirow{2}{*}{ Point 25 } & ERSD & 0 & 0.6670 & 0.1602 & 0.1824 \\
& ersd & 0 & 0.0962 & 0.1892 \\
\hline
\end{tabular}

$$
Y=-0.24 x^{5}+2.57 x^{4}-9.28 x^{3}+12.89 x^{2}-0.88 x+0.14
$$

$$
Y=0.12 z^{5}-0.31 z^{4}-1.47 z^{3}+4.79 z^{2}+1.05 z+0.09
$$

where $Y$ is the relative damage intensity di, $x$ is the mean value of ersd for three tests at different excitation points, and $z$ is the mean value of ERSD for three tests at different excitation points, too.

(23) and (24) can be expressed as two data-fitting curves. The data-fitting curves and test curves are shown in Figure $10(a)$. It is not difficult to verify whether the test curves and fitting curves are nearly overlapped. It is believed that (23) and (24) have a better fitting degree to the quantitative relationship between di and the damage index. Once the damage index is known, di can be calculated reversely. Thus, (23) and (24) can be used to identify the damage intensity of partial damage within the retaining wall. For instance, $\mathrm{di}=5.2$, when ersd $=1$. Thus, $\mathrm{DI}=\mathrm{di} /$ $100=5.2 \%$. Similarly, $\mathrm{di}=4.27$, when $\mathrm{ERSD}=1$. Thus, $\mathrm{DI}=\mathrm{di} / 100=4.27 \%$. Thus, the partial damage intensity of the wall can be identified via this quantitative relationship.

Under the same case, the values of ersd and ERSD are close, when DI is much less (early damage). The value of ERSD becomes much larger than the one of ersd when DI is large, as shown in Figure 10(a). It is predicted that the gap between ERSD and ersd will become larger and larger with continuous increase of DI. Thus, the fluctuation intensity of ERD $(\omega)$ is much larger than the one of er $\mathbf{d}(\bar{\omega})$. To some extent, the damage sensitivity of ERSD is better than that of ersd.

The location of excitation point has certain influence on the damage indexes. The values of damage index will fluctuate when the excitation location changes under the same damage intensity, as shown in Figures 10(b) and 10(c), where the ersd and ERSD are mean values of damage indexes at each excitation point for three vibration tests. To describe this fluctuation intensity of the damage indexes, the variance analyses of the damage indexes are performed under each damage intensity. The variance curves of ersd and ERSD are plotted, as shown in Figures 10(b) and 10(c). With variations of excitation position, the fluctuation intensity of ERSD is lower, but the fluctuation intensity of ersd is much higher. Consequently, the location of excitation point has larger influence on the ersd, but has smaller influence on the ERSD. Thus, the stability of ERSD is better than that of ersd.
Theoretically speaking, both IRF and VIRF are the inherent attributes to retaining wall structures. Both ersd and ERSD, which are based on IRF and VIRF, respectively, should be valid for damages' diagnosis for the retaining wall. However, the validity of ERSD is better than that of ersd. This outcome may be caused by the following factors. (1) The frequency ranges of IRF and VIRF, and the frequency ranges of IRF, are determined by the external excitation and structural responses, and the frequency ranges of VIRF are determined by the structural responses. Especially, the frequency ranges of IRF may be smaller, if the frequency range of the external excitation is not matching well with those of the responses. Due to this, the value of ersd may become smaller. In comparison with IRF, changes of the frequency range of VIRF are smaller, because the frequency ranges of the virtual excitation and virtual response are close. So, the value of the ERSD may be larger. (2) Mode aliasing. The mode aliasing phenomena is caused by such factors as EMD algorithm, frequency components of the original signal, the amplitude of the original signal, and sampling frequency of the original signal. In this paper, the sampling frequency of the original signal and algorithm for EMD are constant. The mode aliasing may be mainly caused by the frequency components and the amplitude of the original signal. Due to the mode aliasing, the IMF component may contain two or more frequency components. The damage sensitivity of the IMF component becomes poor due to the mode aliasing, and the value of the damage index may become smaller. Thus, the mode aliasing should be eliminated to improve the damage sensitivities of IMF components. (3) The order of EMD. The number of bands will become smaller and the value of damage index will become larger, if the order of EMD is smaller. However, the damage information may be lost. The number of bands will become larger, if the order of EMD is larger. With an increase of the band numbers, the number of feature bands may become larger too. This may make the value of the damage index smaller. Thus, only the order of EMD is optimum, the number of the feature bands is the most appropriate. (4) The value of $\varepsilon_{0}$. The $\varepsilon_{0}$ has bigger influences on the value of damage index. The number of feature bands is determined by the $\varepsilon_{0}$. The number of feature bands will become larger, if the value of $\varepsilon_{0}$ is smaller. The value of damage index may become smaller due to the smaller $\varepsilon_{0}$. The number of feature bands will become smaller, if the value of $\varepsilon_{0}$ is larger. Although the value of damage index may become larger, the damage information may be lost. Thus, the gap between ersd 


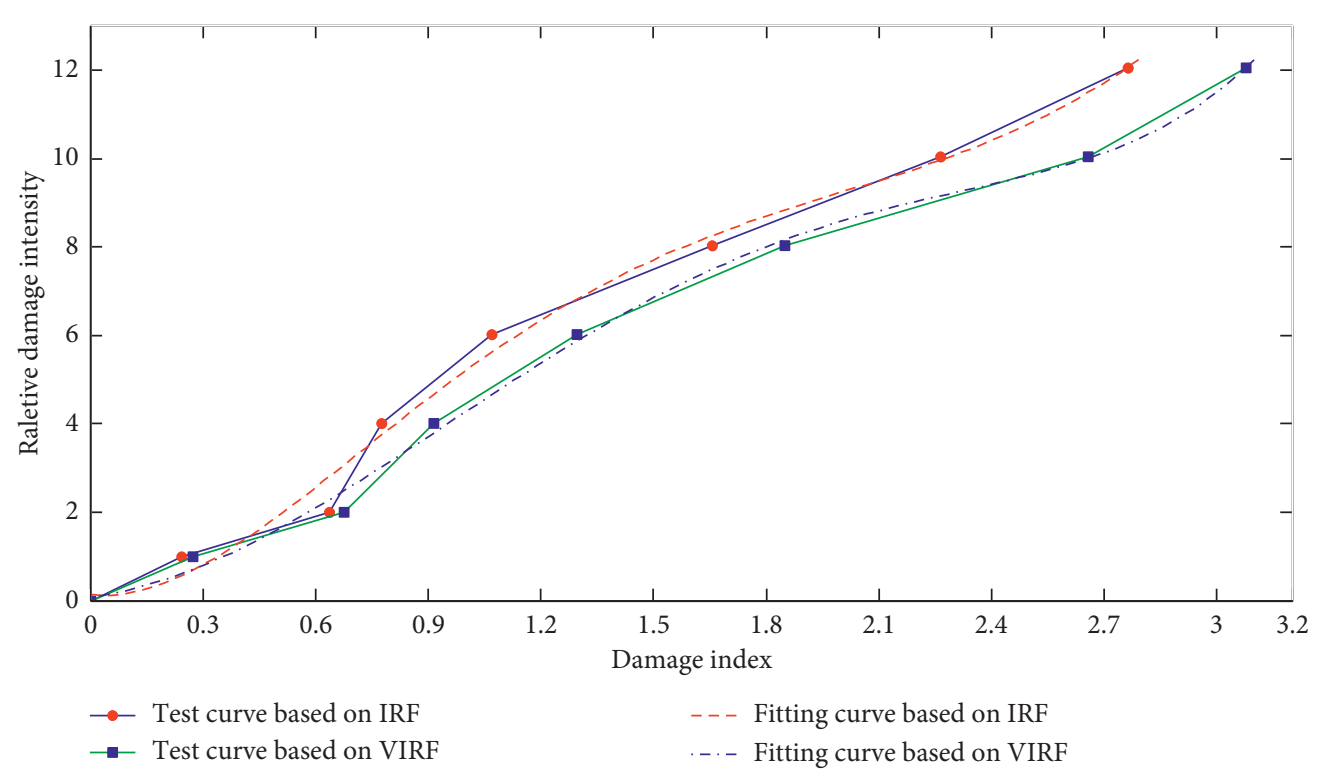

(a)

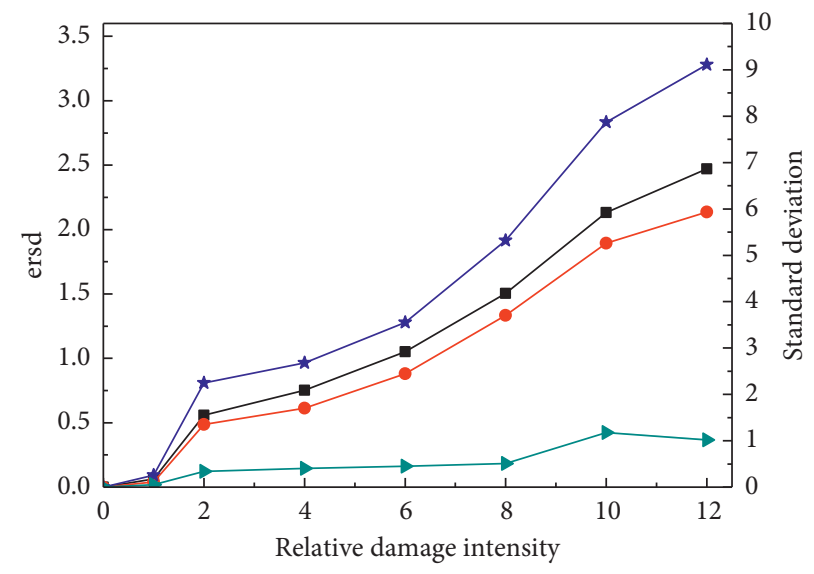

$\rightarrow$ Excitation point I

$\rightarrow$ Excitation point II

$\star$ Excitation point III

$\rightarrow$ Curve of standard deviation

(b)

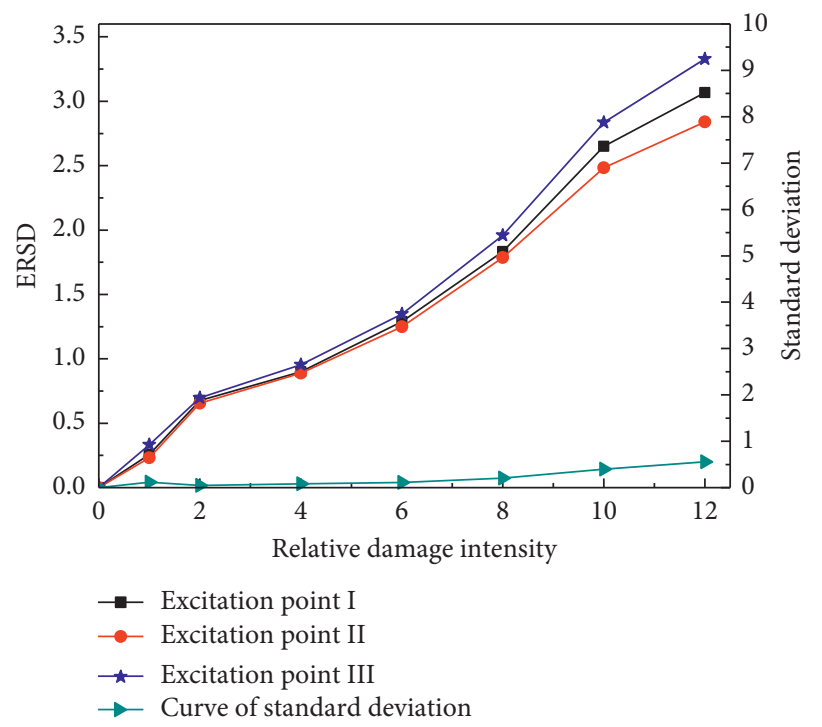

(c)

FIGURE 10: Relationship curve between the damage index and damage intensity. (a) Tests curves and fitting curves. (b) Curves based on IRF. (c) Curves based on VIRF. 
and ERSD may be related to the $\varepsilon_{0}$. (5) The coherence of data. The mutual interference of structural responses exists in the structural system. Either IRF or VIRF is affected by the mutual interference of responses. The value of the damage index is also affected by the coherence of data.

\section{Conclusions}

In this paper, two damage indexes ersd and ERSD are proposed via the Hilbert-Huang Transforms of IRF and VIRF. Damages within the pile plate retaining wall are identified via ersd and ERSD. The damage state of the wall can be detected sensitively, the damage location can be diagnosed validly, and the damage intensity can also be identified quantitatively via ersd and ERSD. Similarly, ersd and ERSD can also be used to identify the damages within other types of retaining walls (such as gravity retaining walls, cantilever retaining walls). Although only the single damage within the wall is diagnosed via ersd and ERSD, the multidamages within the wall can also be diagnosed similarly. In addition, ERSD has much better stability, but ersd has much poorer stability. Besides, the damage sensitivity of ERSD is better than that of ersd. Thus, the outcomes of damage identification of ERSD are much better than the ones of ersd.

Due to the difference of damage sensitivity to different measuring points and the requirements for locating damage, enough measuring points should be fixed on the wall to diagnose damages sensitively and validly. In addition, the location of partial damages can be precisely identified when sensors are very close to partial damages. However, the damage location is unknown in practice. Thus, it is required that sensor locations should be adjusted repeatedly to diagnose the location of partial damage precisely. In addition, the identification of damage intensity is related to tests data. The tests data are much more, and the identification accuracy of damage intensity is much better. Thus, the precise identification of damage intensity requires the collection of a great deal of tests data. Besides, the threshold value $\varepsilon_{0}$ has great influence on extracting feature bands. Thus, the investigation of $\varepsilon_{0}$ should be performed to select reasonable feature bands in the future.

In the literature [29], a damage identification method for retaining walls is proposed based on the Wavelet Packet frequency band energy spectrum. The feature bands, which are used to detect damage, are the ones with much larger energy. In the literature [24], a damage diagnosis method for retaining walls is also proposed based on the Hilbert energy spectrum. The feature bands are the ones with much larger energy too. In these two methods, it is thought that the bands with larger energy are useful for damage identification without considering damage sensitivity of bands. However, the damage sensitivities of those bands with larger energy may be not strong, and the sensitivities of those bands with smaller energy may not be poor. In this paper, the feature bands are determined by the damage sensitivities of bands. Those bands with stronger damage sensitivities are extracted to identify damage. It may be much more reasonable to select feature bands via damage sensitivity of bands.
Due to some technology issue, the Hilbert-Huang Transforms of IRF and VIRF were performed without considering aliasing and leakage phenomena. And, the analysis of coherence in experimental data is also ignored. However, the aliasing, leakage phenomena, and coherence in data should be performed to get more precise results. In addition, all the order of EMD is ten in this paper. However, the optimum order of EMD to IRF or VIRF may be variable. Thus, the optimum order of EMD needs to be investigated. Besides, the value of $\varepsilon_{0}$ is also variable. To select the valid feature bands, the investigation of the optimum value of $\varepsilon_{0}$ needs to be done. Thus, these works should be considered in the future.

\section{Data Availability}

The data used in this paper are available within this paper. Based on the tables and figures in this paper, the readers can verify the results of this paper, replicate the analysis, and conduct secondary analyses.

\section{Conflicts of Interest}

The author of this paper does not have any conflicts of interest regarding the publication of this paper.

\section{Acknowledgments}

This paper was finalized by National Natural Science Foundation of China (No. 51908345), Key Program of Social Development by Shaanxi Provincial Science and Technology Department (No. 2020SF-430), and Scientific Research Program Funded by Shaanxi Provincial Education Department (I7JK0157 and 20JK0559).

\section{References}

[1] S. W. Doebling, C. R. Farrar, and M. B. Prime, "A summary review of vibration-based damage identification methods," The Shock and Vibration Digest, vol. 30, no. 2, pp. 91-105, 1998.

[2] G. Hearn and R. B. Testa, "Modal analysis for damage detection in structures," Journal of Structural Engineering, vol. 117, no. 10, pp. 3032-3063, 1991.

[3] M. Biswas, A. K. Pandey, and M. M. Samman, "Diagnostic experimental spectral/modal analysis of highway bridge," International Journal of Analytical and Experimental Modal Analysis, vol. 5, no. 1, pp. 33-42, 1990.

[4] C. Zhang, G. Q. Song, and G. Y. Wu, "Structural damage identification by synchronal updating measured modes and finite element model," Journal of Vibration and Shock, vol. 29, no. 9, pp. 1-4, 2010.

[5] Z. H. Zong, F. P. Zhu, and J. Niu, "Damage identification method of bridge structures using response surface based on finite element model updating," China Civil Engineering Journal, vol. 46, no. 2, pp. 115-122, 2013.

[6] R. Perera and R. Torres, "Structural damage detection via modal data with genetic algorithms," Journal of Structural Engineering, vol. 132, no. 9, pp. 1491-1501, 2006.

[7] M. Varmazyar, N. Haritos, and M. Kirley, "A Bayesian damage identification technique using evolutionary 
algorithms-a comparative study," Electronic Journal of Structural Engineering, vol. 14, no. 1, pp. 1-19, 2015.

[8] W. L. Qu and W. Chen, "Seismic damage diagnosis of frame structure using artificial neural network," Earthquake Engineering and Engineering Vibration, vol. 22, no. 1, pp. 43-48, 2002.

[9] B. S. Wang, C. B. Liu, and G. B. He, "Detection for existence of structure damage using statistical neural network," China Civil Engineering Journal, vol. 37, no. 8, pp. 24-27, 2004.

[10] Z. Hou, M. Noori, and R. S. Amand, "Wavelet-based approach for structural damage detection," Journal of Engineering Mechanics ASCE, vol. 126, no. 7, pp. 677-683, 2000.

[11] J. Li and X. Fan, "Structural damage identification with extract impulse response function and optimal sensor location," Electronic Journal of Structural Engineering, vol. 14, no. 1, pp. 123-132, 2015.

[12] Y. L. Ding, A. Q. Li, and T. Liu, "A study on the WPT-based structural damage alarming of the ASCE benchmark experiments," Advances in Structural Engineering, vol. 11, no. 1, pp. 121-127, 2008.

[13] J. D. Zheng, Z. S. Sun, and W. X. Ren, "Improved HilbertHuang transform and its application to rolling bearing fault diagnosis," Journal of Mechanical Engineering, vol. 51, no. 1, pp. 138-145, 2015.

[14] Z. N. Huang and B. Hu, "BFGS perturbation method for damage detection of fixed- fixed beam," Journal of Vibration, Measurement \& Diagnosis, vol. 36, no. 3, pp. 536-540, 2016.

[15] Y. Z. Fu, Z. R. Lu, and J. K. Liu, "Damage identification in plates using finite element model updating in time domain," Journal of Sound and Vibration, vol. 332, no. 26, pp. 70187032, 2013.

[16] X. Z. Zhang, W. J. Yao, B. Chen, and D. Liu, "Damage identification of a pile based on vibration characteristics," Science World Journal, vol. 2014, pp. 1-8, Article ID 150516, 2014.

[17] H. Li, "The application of improved genetic algorithm on damage identification for frame structure," International Journal of Control and Automation, vol. 9, no. 2, pp. 229-238, 2016.

[18] Y. L. Zhao, J. K. Liu, Z. R. Lv et al., "Structural damage identification based on residual vectors and Tree-seed algorithm," Acta Scientiarum Naturalium Universitatls Sunyatseni, vol. 56, no. 4, pp. 46-50, 2017.

[19] Y. B. Jiao, H. B. Liu, Y. C. Cheng, and Y. F. Gong, "Damage identification of bridge based on Chebyshev polynomial fitting and fuzzy logic without considering baseline model parameters," Shock and Vibration, vol. 2015, pp. 1-10, Article ID 187956, 2015.

[20] H. B. Liu, X. Q. Wang, Y. F. Gong, and Y. Jiao, "Damage identification of urban overpass based on hybrid neurogenetic algorithm using static and dynamic properties," Mathematical Problems in Engineering, vol. 2015, pp. 1-10, 2015.

[21] Q. Xu, "Investigation of stability alarming for retaining wall structures with damage," Shock and Vibration, vol. 2017, pp. 1-12, 2017.

[22] Y. Li, "On-line structural health monitoring offshore structures based on wavelet analysis," Ocean Engineering, vol. 34, no. 5, pp. 131-136, 2016.

[23] Y. Liu and A. Q. Li, "Experimental study on damage identification of the benchmark structure under ambient excitation," Chinese Journal of Computational Mechanics, vol. 27, no. 3, pp. 500-504, 2010.

[24] Q. Xu, "Investigation of damage diagnosis of retaining wall structures based on the Hilbert damage feature vector spectrum," Shock and Vibration, vol. 2019, pp. 1-23, Article ID 3509470, 2019.

[25] N. E. Huang, Z. Shen, S. R. Long et al., "The empirical mode decomposition and the Hilbert spectrum for nonlinear and non-stationary time series analysis," Proceedings of the Royal Society of London. Series A: Mathematical, Physical and Engineering Sciences, vol. 454, no. 1971, pp. 903-995, 1998.

[26] N. E. Huang, M. L. C. Wu, S. R. Long et al., "A confidence limit for the empirical modedecomposition and Hilbert spectral analysis," Proceedings of the Royal Societyof London. Series A: Mathematical, Physical and Engineering Sciences, vol. 459, no. 2037, pp. 2317-2345, 2003.

[27] C. X. Shi and H. S. Li, "Experimental study on damage detection of a girder bridge based on Hilbert marginal spectrum and random-fuzzy statistics principle," Journal of Vibration and Shock, vol. 30, no. 8, pp. 123-127, 2011.

[28] C. X. Shi, Hilbert-huang Transform and its Application in Structural Analysis, Tongji University, Shanghai, China, 2004.

[29] Q. Xu, "Damage identification investigation of retaining wall structures based on a virtual impulse response function," Shock and Vibration, vol. 2016, no. 12, pp. 1-13, Article ID 1346939, 2016.

[30] H. D. Xu, L. H. Wang, and Z. D. Hu, "Quantitative identification of wood beam damage using modal curvature theory," Journal of Vibration, Measurement \& Diagnosis, vol. 31, no. 1, pp. 110-114, 2011.

[31] H. Y. Zhang, D. Han, F. Wang et al., Hilbert-huang Transform and its Application, p. 311, National Defense Industry Press, Beijing, China, 2017. 